\title{
Simulation of total absorption dual readout calorimetry
}

\author{
principles and performance
}

"14 $14^{\text {th }}$ International Conference on Calorimetry in High Energy Physics", 10-14 May 2010

\section{Hans Wenzel}

Fermilab 술

$11^{\text {th }}$ May 2010

FERMILAB-SLIDES-10-001

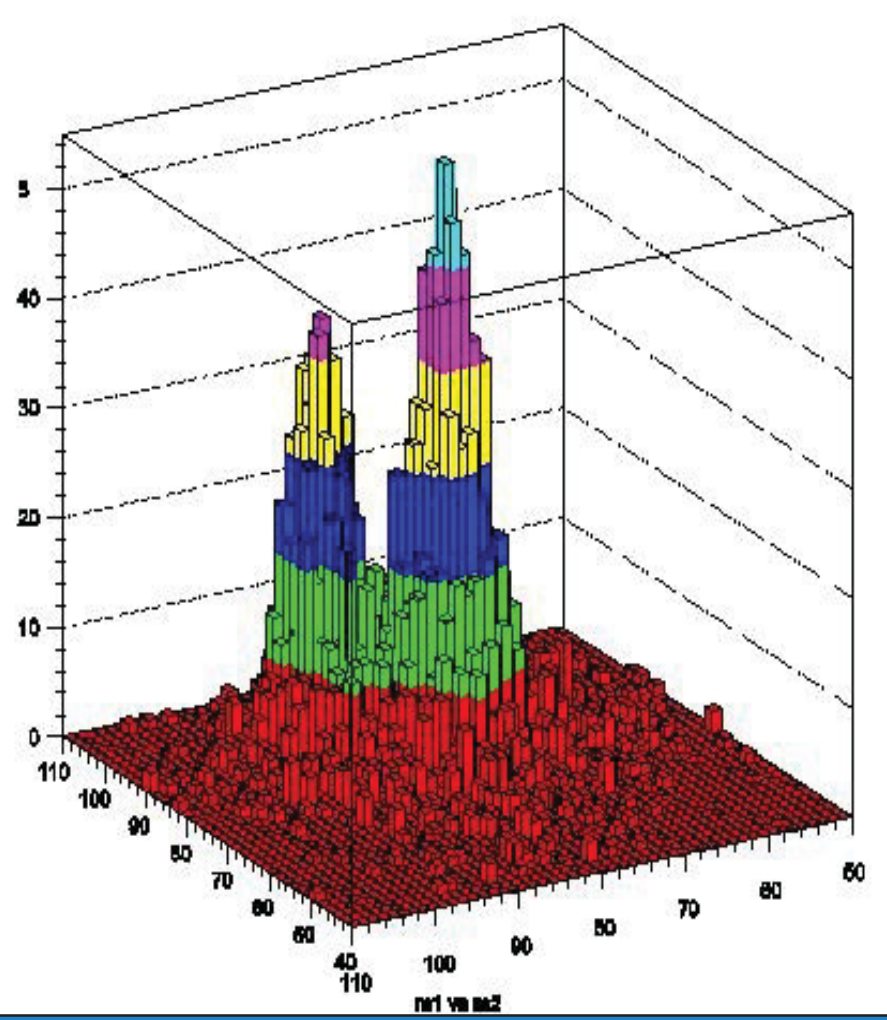




\section{Outline}

Motivation

Principle of a dual read out calorimeter

The software environment:

SLIC

CrystalSim

- The ccal02 detector.

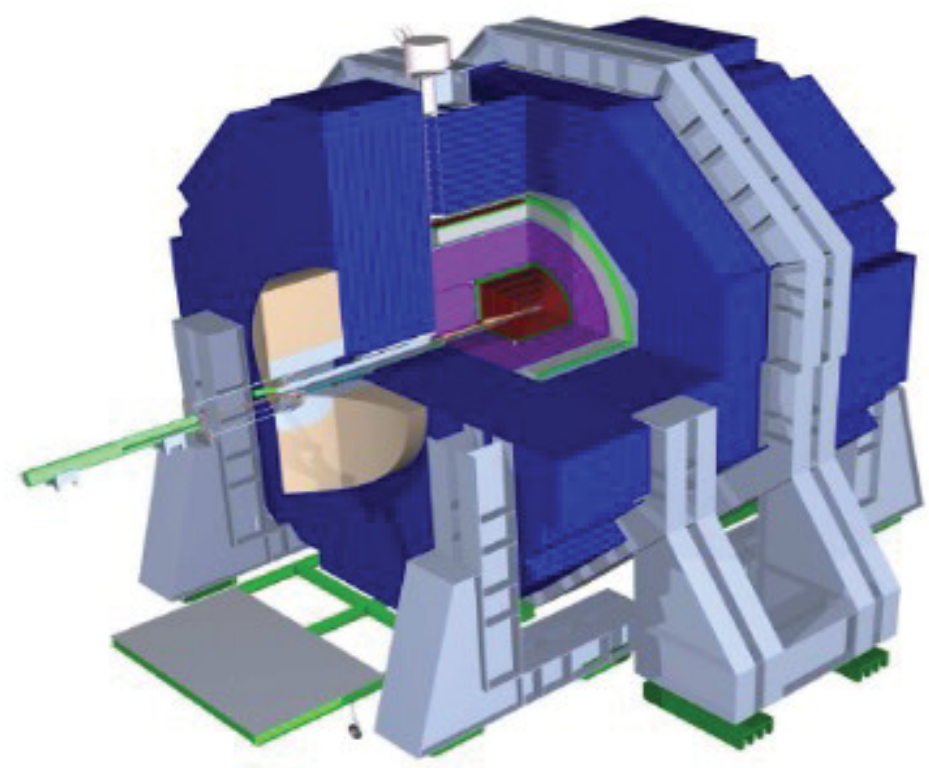

- Analysis:

Calibration using electrons.

Obtaining the dual read out correction.

Effects limiting the resolution

- Modeling of hadronic showers

(can we trust Geant4?)

- Photon statistic (in progress)

- leakage

- energy dependence of dual read out correction

- Birks attenuation

Resolution for single $\pi$

- Conclusions 


\section{Motivation}

Be ready for all potential physics scenarios we might encounter at a future lepton collider

Totally active dual read out crystal calorimeter:

- Excellent EM calorimeter.

- Excellent hadron calorimeter:

- Totally active, not a sampling calorimeter, even large sampling fraction induces significant stochastic term (dependent on particle type).

- Dual read out.

- $\quad$ Longitudinal segmentation helps to detect and correct for leakage.

- While not a PFA calorimeter, segmentation is fine enough so that particle flow algorithms can be applied.

- Dense scintillating crystals and new economical photo detector like SiPMT are what makes this possible. 


\section{Principle of a dual read out calorimeter}

- $\quad$ Detect separately scintillation and Cerenkov light (same Volume)

- Scintillation light is a precise measure of the total energy released in the calorimeter ( total path length of the charged particles in a shower).

- $\quad$ Cerenkov light is a precise measure of the total path length of the relativistic particles $(\beta>1 / n)$ in the shower.

- $\quad$ Calibrate $\mathrm{C}=\mathrm{S}$ for electron showers (spread of both signals very small)

- Hadron showers with large C/S --> large electromagnetic component, small missing energy.

- Hadron showers with low C/S --> purely charged hadrons, large amount of missing energy.
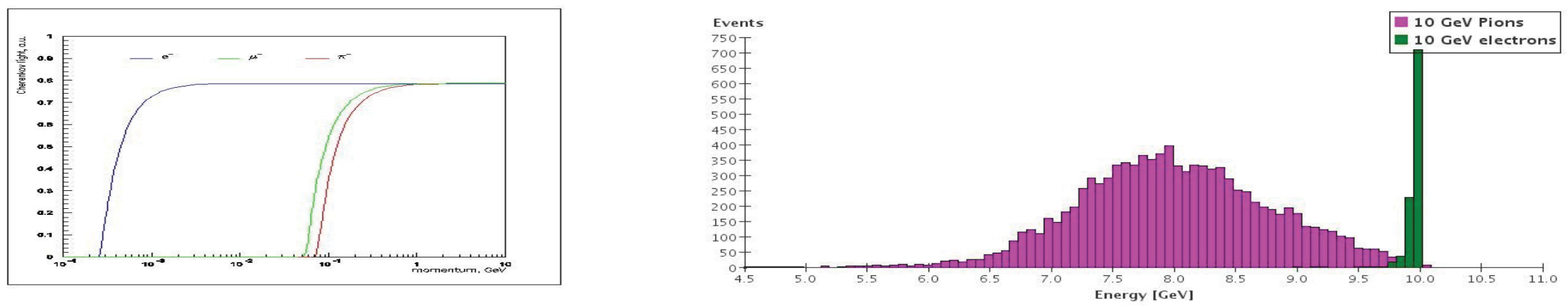


\section{The software environment:}

\section{CCAL02:}

SLIC: Geant 4 based framework for detector simulation.

XML based detector description (geometry and sensitive detectors):

Easy to implement various detector variations: test beam/collider detector, materials, density, em and had segmentation, optical properties, ...

Executing a simple script creates all files necessary for simulation and analysis. Variety of physics lists.

LCIO event output

Using SLI C allows us to make use of the entire SID framework:

SLIC $(C++)$, Icsim.org (netbeans), WIRED, JAS3, LCIO Event Browser (JAVA) ..... $\rightarrow$ this allows us to study physics performance as part of a complete detector. (Very nice development environment.)

If one prefers ROOT we have a LCIO to root converter for the relevant Icio classes. Easy to run SLIC on the grid $\rightarrow$ we have Grid scripts: make it easy to generate large data sets, takes care of names, random seeds etc.,

http://confluence.slac.stanford.edu/display/ilc/How+do+I+use+the+OSG+Grid

Calibration and analysis is automated using the Icsim.org 


\section{What needed to be done to simulate total absorption dual read out calorimeter in SLIC}

Need to add optical physics (Cerenkov,Scintillation etc.,) $\rightarrow$ now can be used with any physics list.

- Need to be able to add optical properties to materials in the detector description e. g. refraction index/absorption as function of photon energy.

- Implement Birk's suppression

- $\quad$ Sensitive detector needs to be able to produce multiple hit Collections (Energy deposition, Cerenkov) $\rightarrow$ this is allowed in GEANT 4 but SLIC in its original form only allowed for one Hit collection per sensitive collector.

- Implement special optical calorimeter class:

- Register energy deposition (Edep hits).

- deal with optical photons. We don't track optical photons but kill them after the first step and add their energy to the Cerenkov hits. 


\section{CrystalSim: photon statistics and timing etc.}

$\rightarrow$ Geant 4 based stand alone application

$\rightarrow$ tracks every optical photon from time of production until it's lost (absorbed) or detected at the photo-sensors.

$\rightarrow$ Input: rindex $(\lambda)$, absorption length $(\lambda)$, scintillation $\operatorname{spectrum}(\lambda, t)$, Birks suppression, crystal surface conditions.

$\rightarrow$ Since geant 4.9.3 LUT exist which describe various surface types (polished, painted, tyvek wrapped..) as measured by a group from LBNL. $\rightarrow$ Quantum efficiency $(\lambda)$ and electronic response is applied in the analvsis step (ROOT).

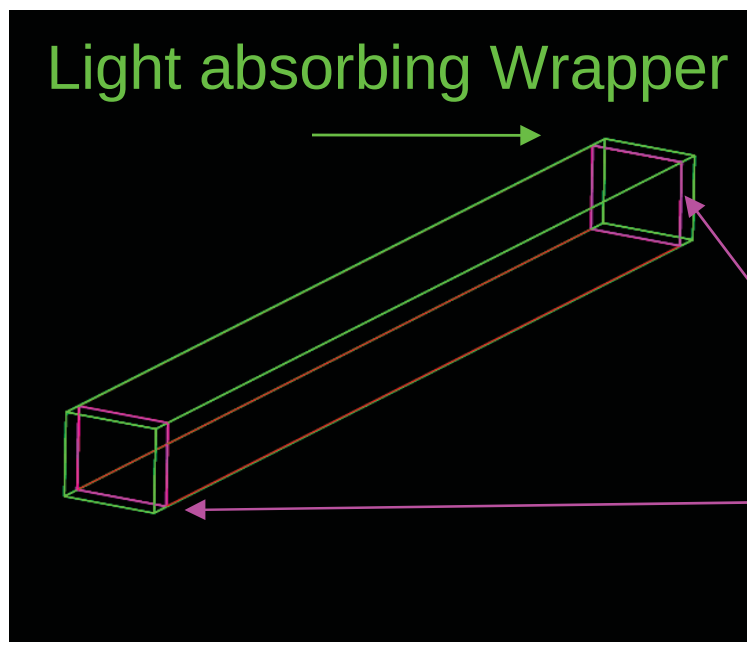

Crystal: 2x2×20 cm

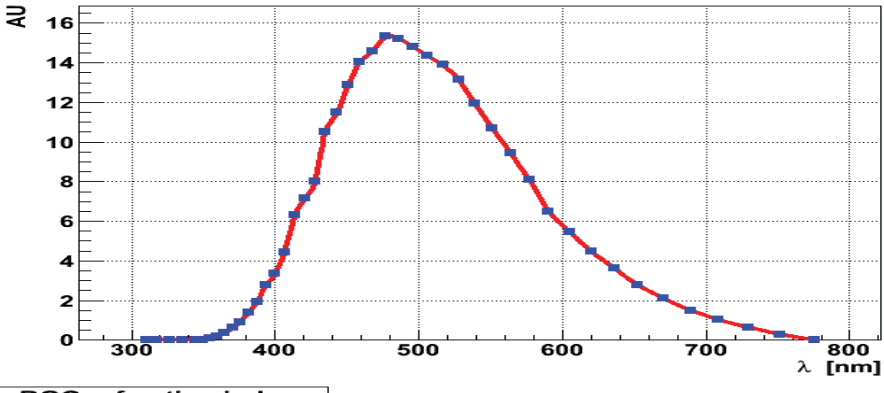

Ideal Photodetectors

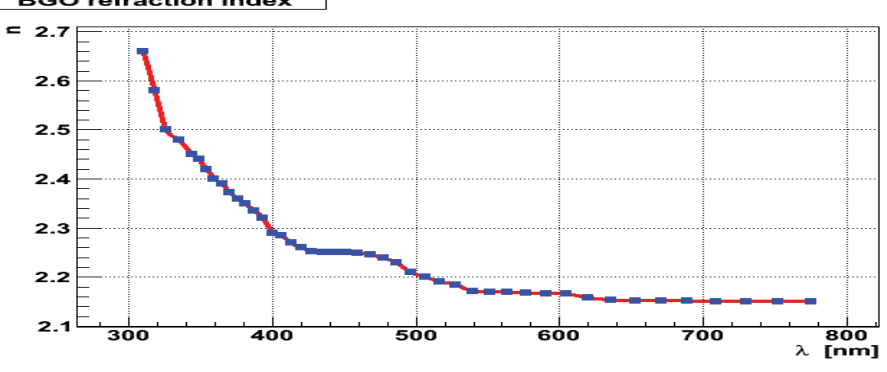




\section{CrystalSim: light at the photo detectors}
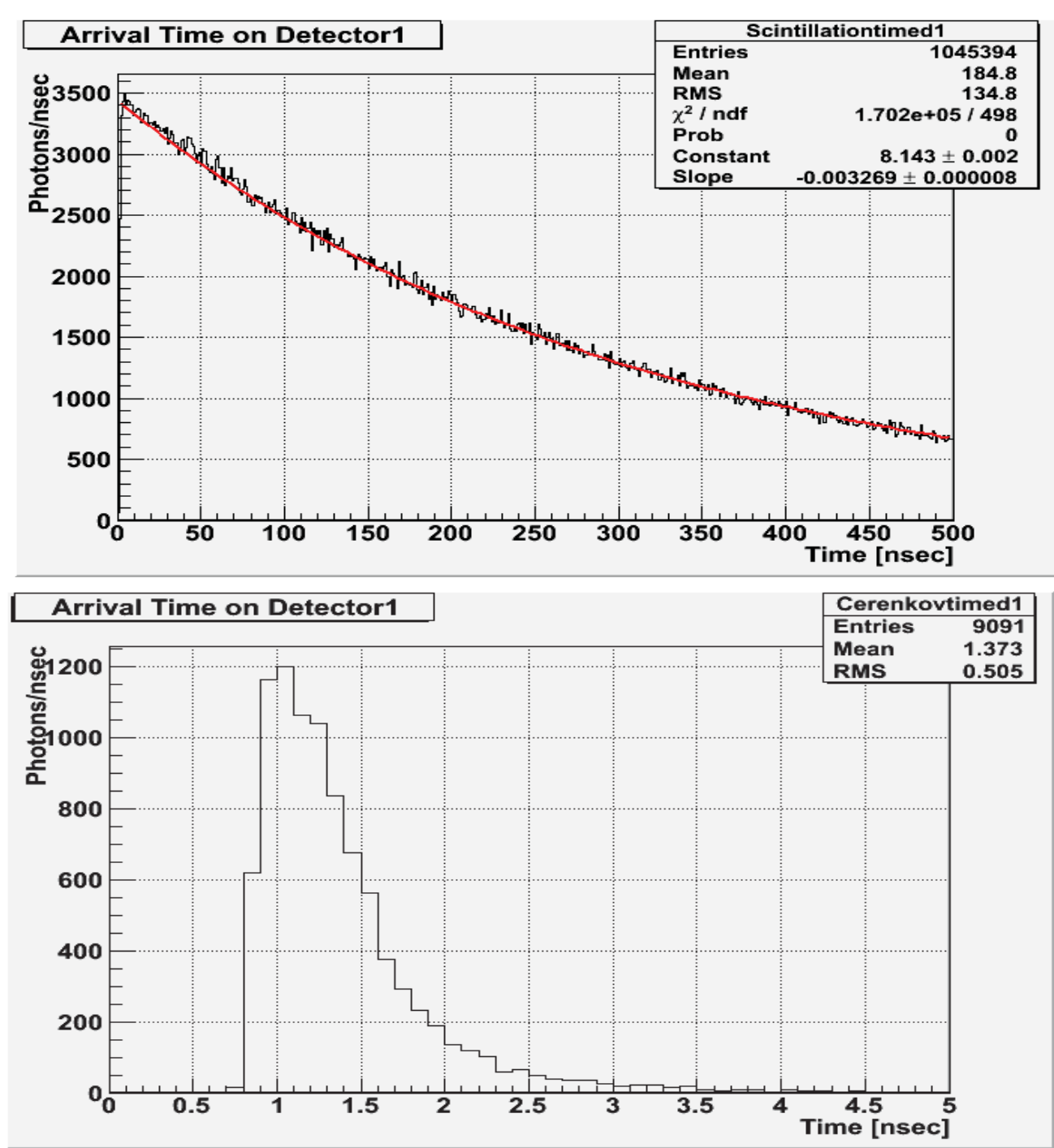

$2 \mathrm{GeV}$ Muons (2cm BGO):

10500: Szintillation ph/sensor 90: Cerenkov ph/sensor

Will provide input to study effects of photostatistics, study how well we can separate cerenkov and scintilation light etc..... 


\section{The CCAL02 detector (Crystal Calorimetry version of SID)}

\begin{tabular}{|c|c|c|c|c|c|c|c|}
\hline \multirow[b]{2}{*}{ Name } & \multirow[b]{2}{*}{ Layers } & \multirow[b]{2}{*}{ Thickness/Layer } & \multirow[b]{2}{*}{ Segmentation } & \multicolumn{2}{|c|}{ BGO } & \multicolumn{2}{|c|}{$\mathrm{PbWO}_{4}$} \\
\hline & & & & $X_{0}$ & $\lambda_{1}$ & Xo & II \\
\hline & & {$[\mathrm{cm}]$} & {$[\mathrm{cm} \times \mathrm{cm}]$} & & & & \\
\hline ECAL Barrel & 8 & 3 & $3 \times 3$ & 21.4 & 1.1 & 27 & 1.3 \\
\hline HCAL Barrel & 17 & 6 & $5 \times 5$ & & 4.7 & & 5.7 \\
\hline Total Barrel & 25 & & & & 5.8 & & 7 \\
\hline ECAL Endcap & 8 & 3 & $3 \times 3$ & 21.4 & 1.1 & & 1.3 \\
\hline HCAL Endcap & 17 & 6 & $5 \times 5$ & & 4.7 & & 5.7 \\
\hline Total Endcap & 25 & & & & 5.8 & & 7 \\
\hline
\end{tabular}

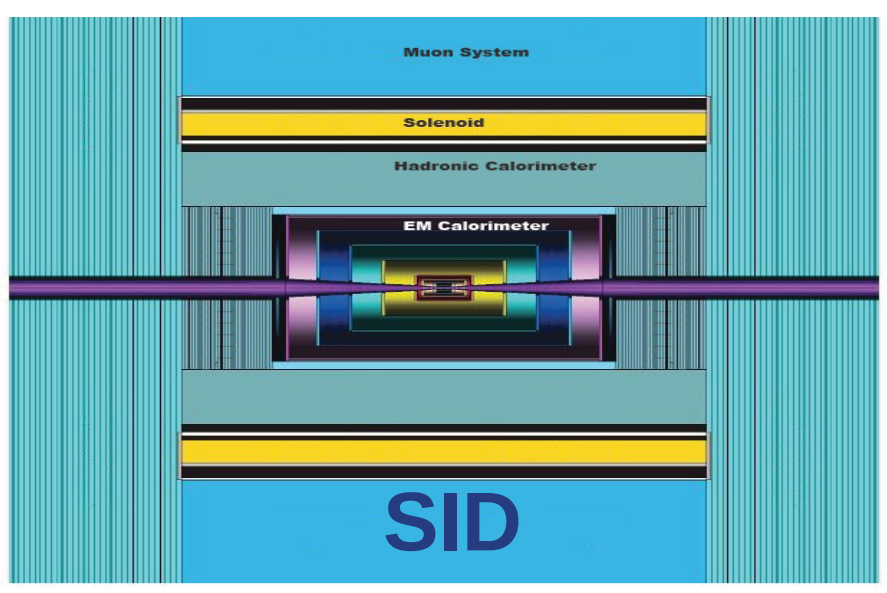

\begin{tabular}{|c|c|c|c|}
\hline Material & Density & Rad. len. XO & IA len. \\
\hline & {$[\mathrm{g} / \mathrm{cm} 3]$} & {$[\mathrm{cm}]$} & {$[\mathrm{cm}]$} \\
\hline BGO & 7.13 & 1.12 & 21.88 \\
\hline PbWO4 & 8.3 & 0.9 & 18 \\
\hline SCG1-C & 3.36 & 4.25 & 45.6 \\
\hline
\end{tabular}

Mönté Carlo: BGO with $15.0 \mathrm{~g} / \mathrm{cm}^{3}$ 


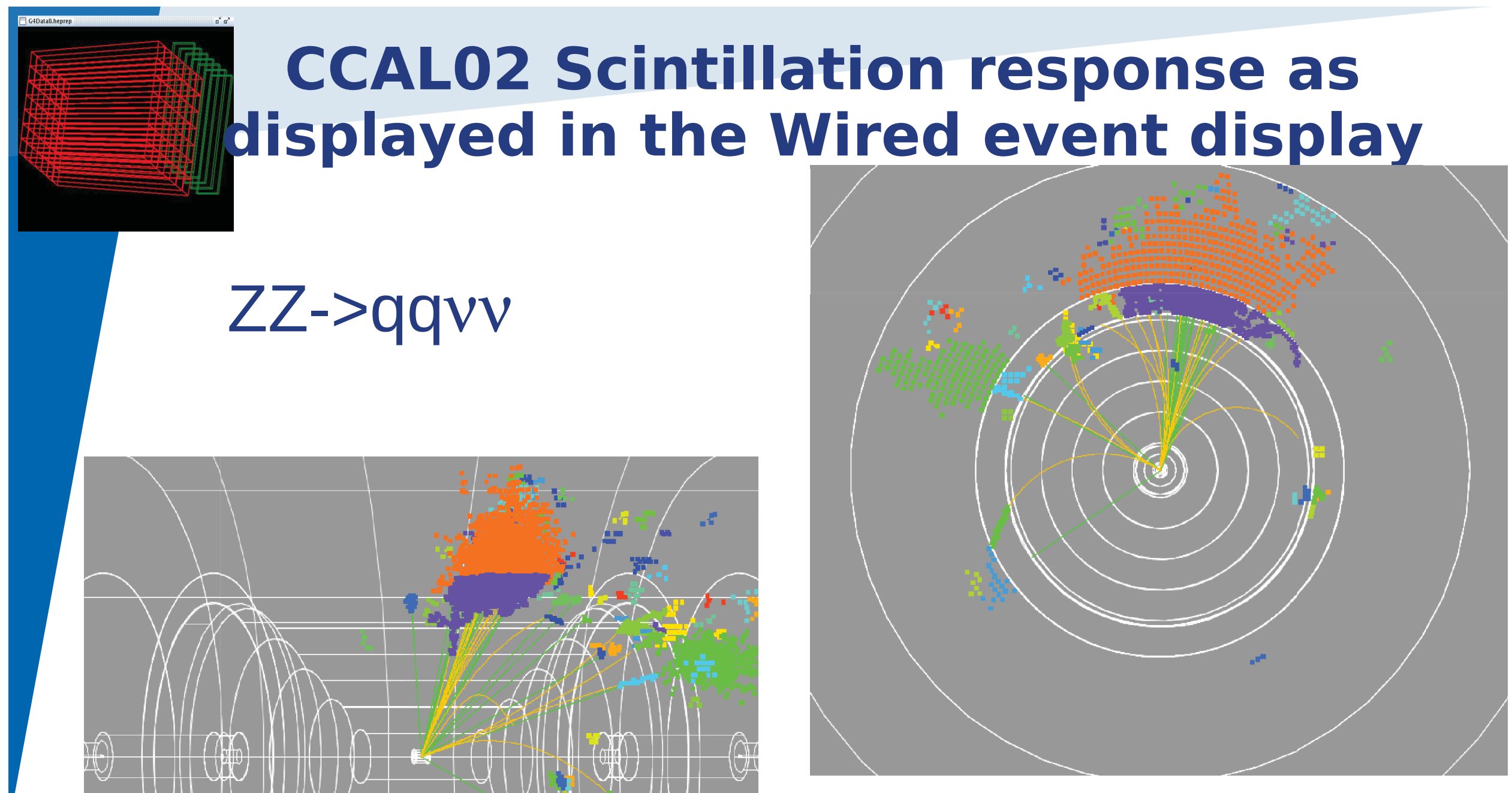

Digisim 


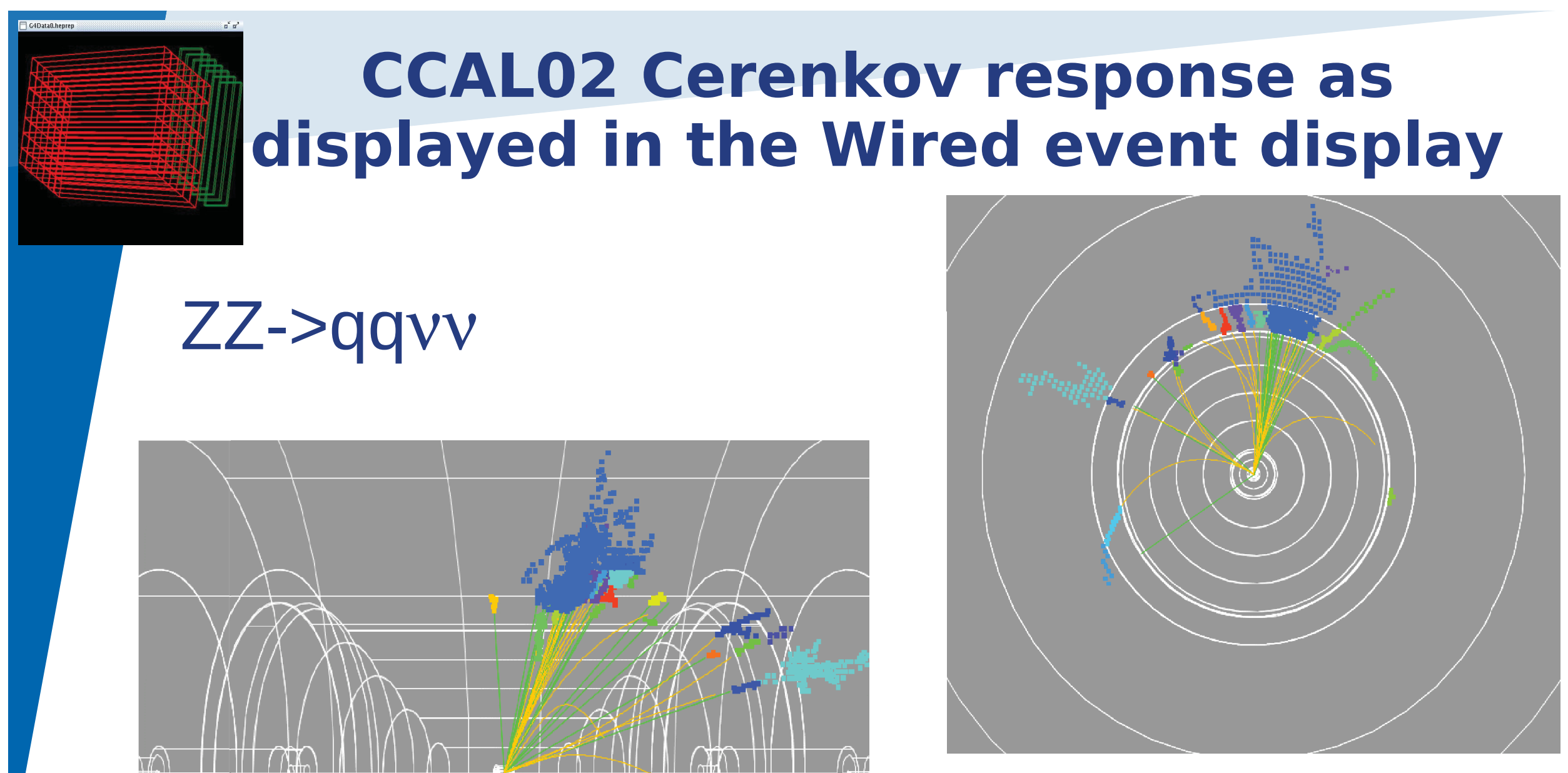




\section{Electron Calibration for Scintillator, Cerenkov}

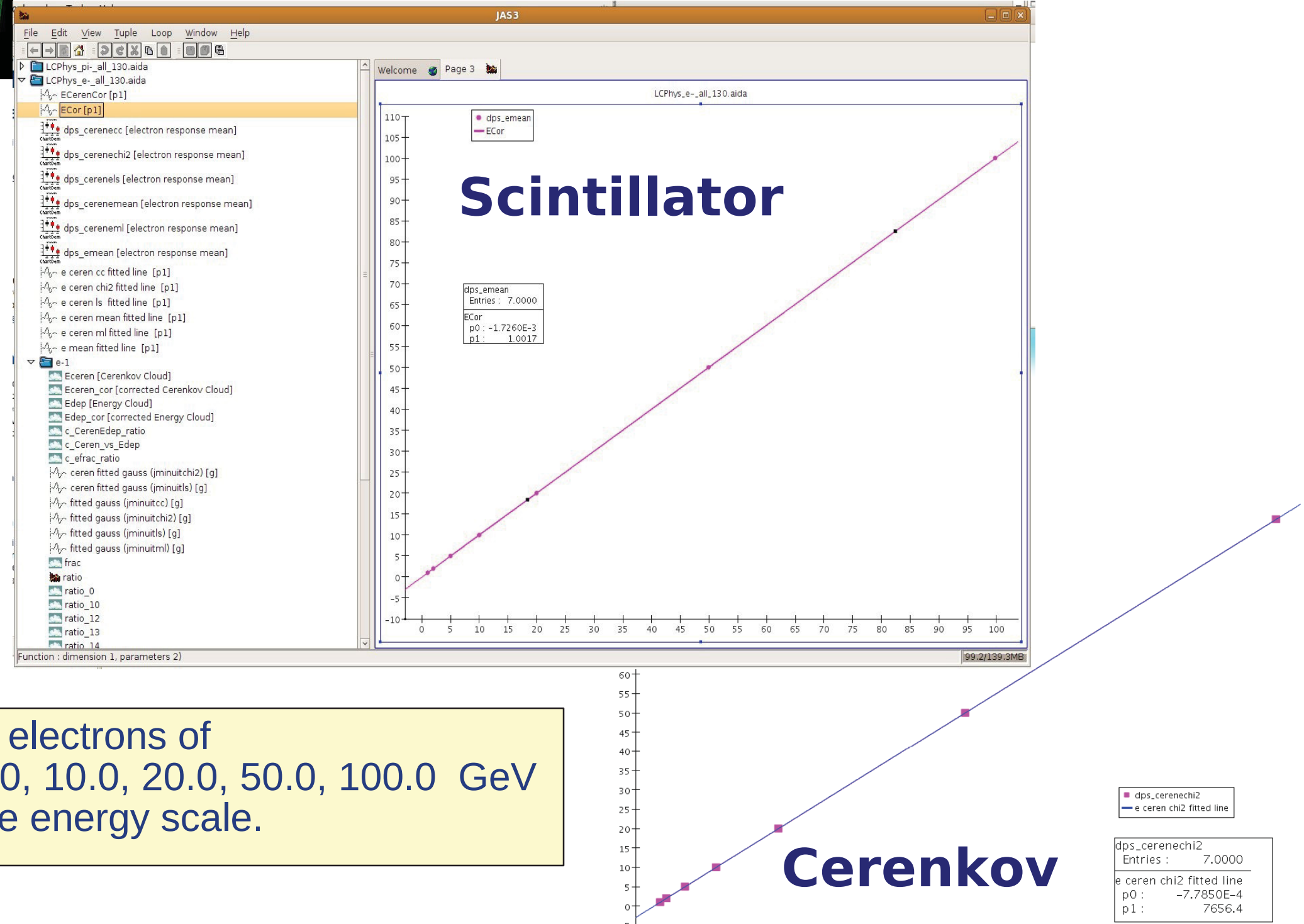

Use single electrons of

1.0, 2.0, 5.0, 10.0, 20.0, 50.0, $100.0 \mathrm{GeV}$ To estimate energy scale. 


\section{Analysis: Electron Calibration for Scintillator, Cerenkov}
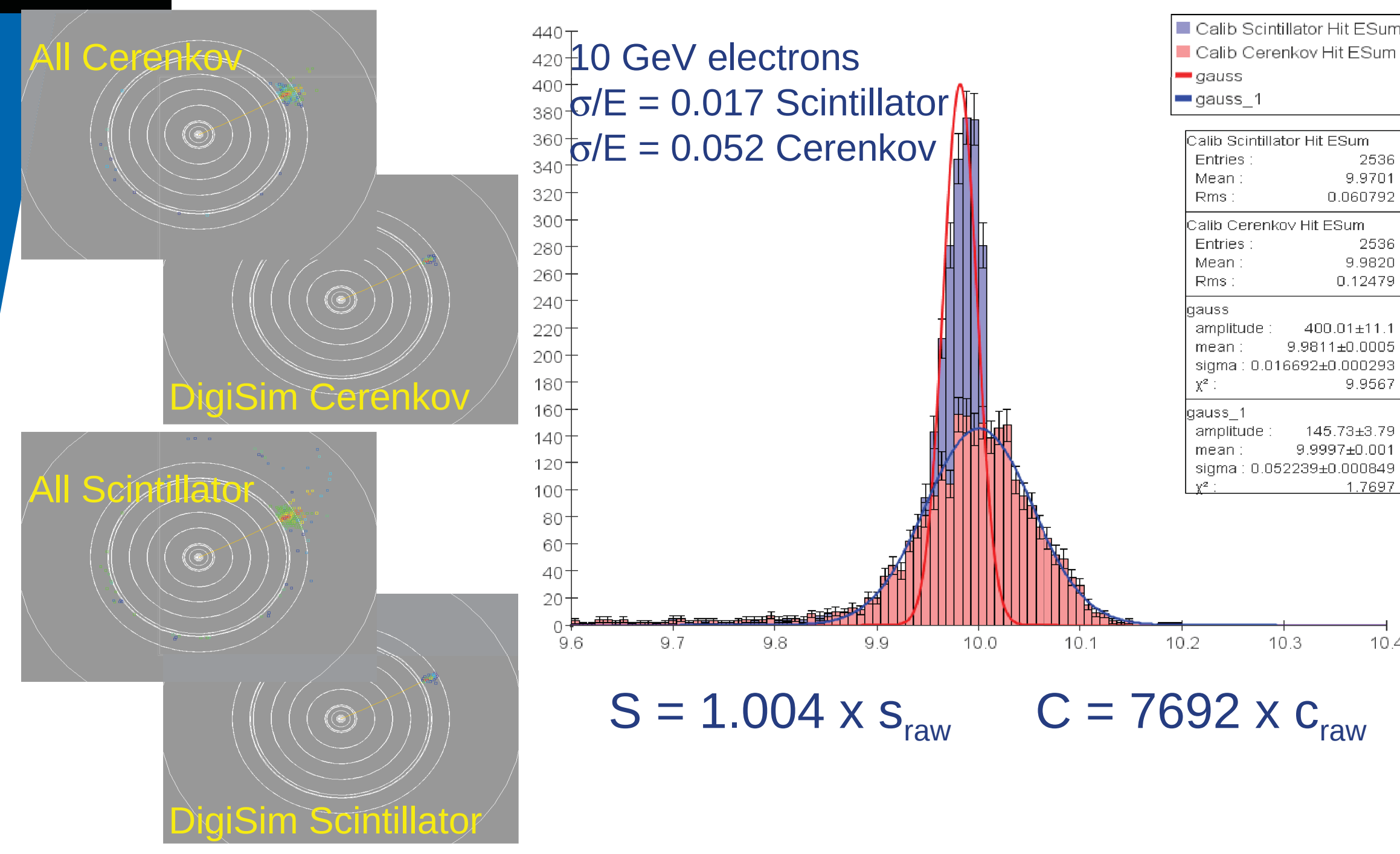


\section{Polynomial Correction Functions: $\mathbf{E}=\mathbf{S} / \mathbf{P n}$}

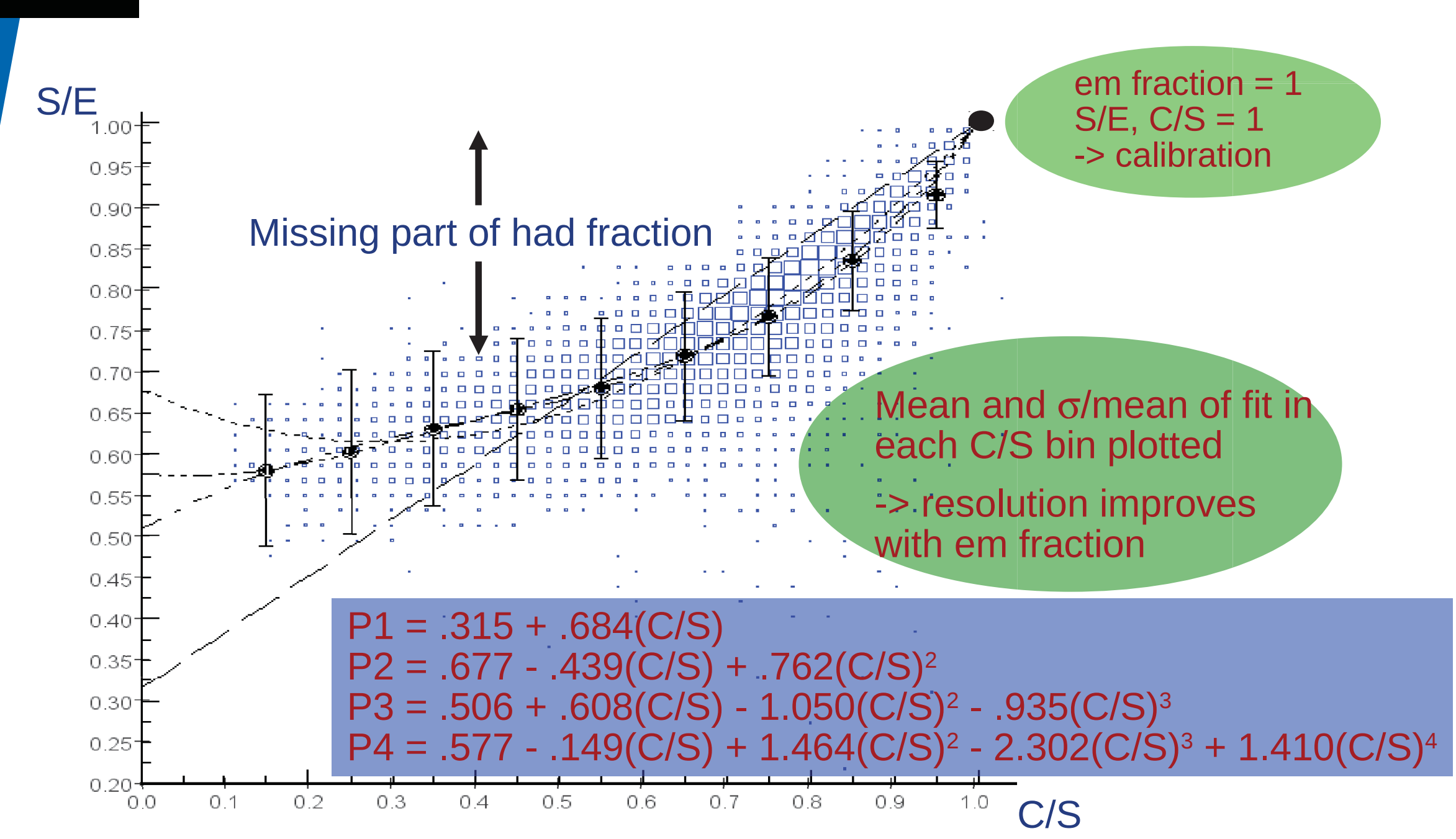




\section{Correction function as function of energy}

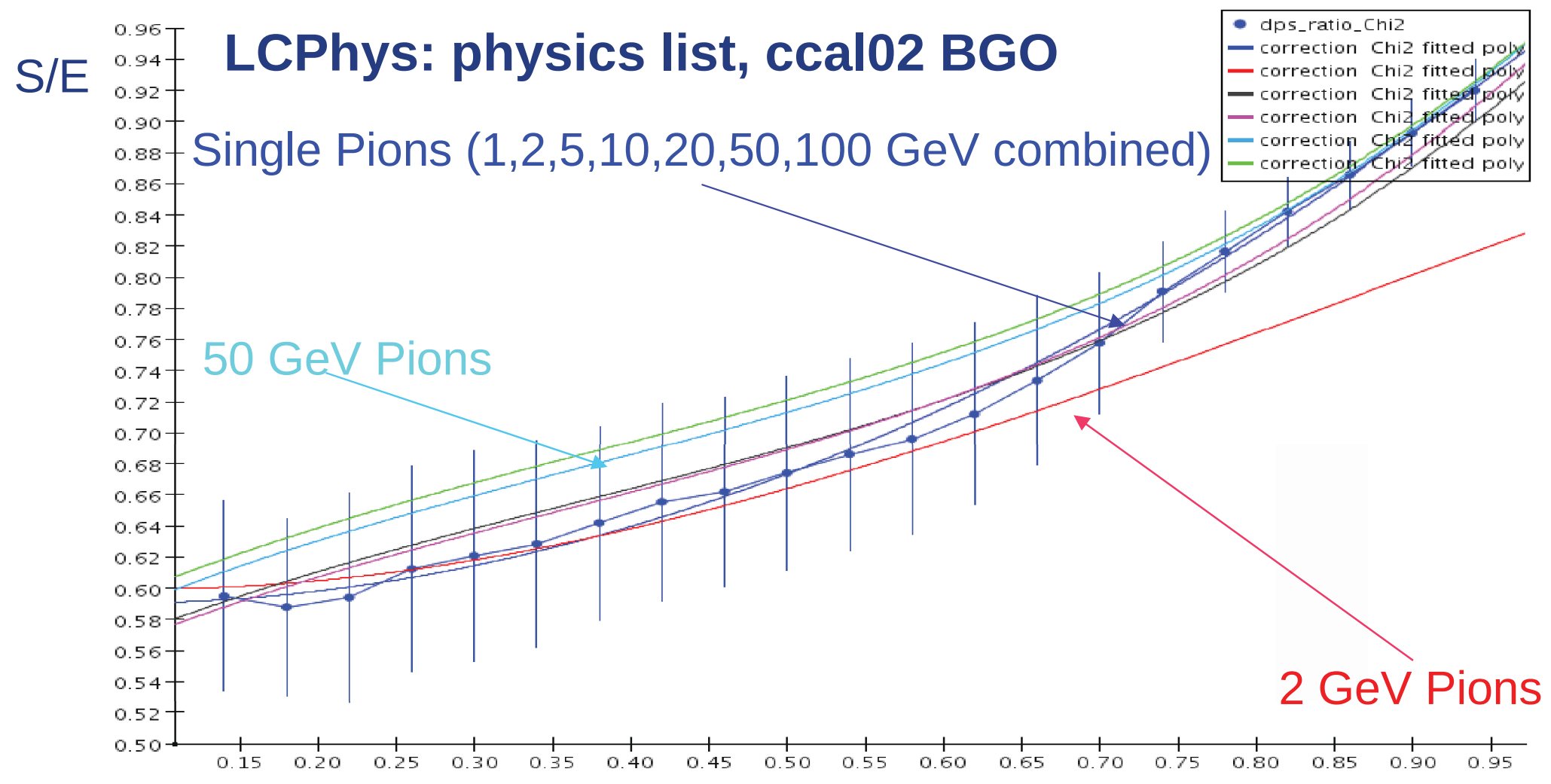

Note! Dual read out correction almost independent of energy, $\mathrm{C} / \mathrm{S}$ but it's worth exploring if we can improve energy resolution with energy dependent correction function 


\section{Corrected single $\pi^{-}$response}

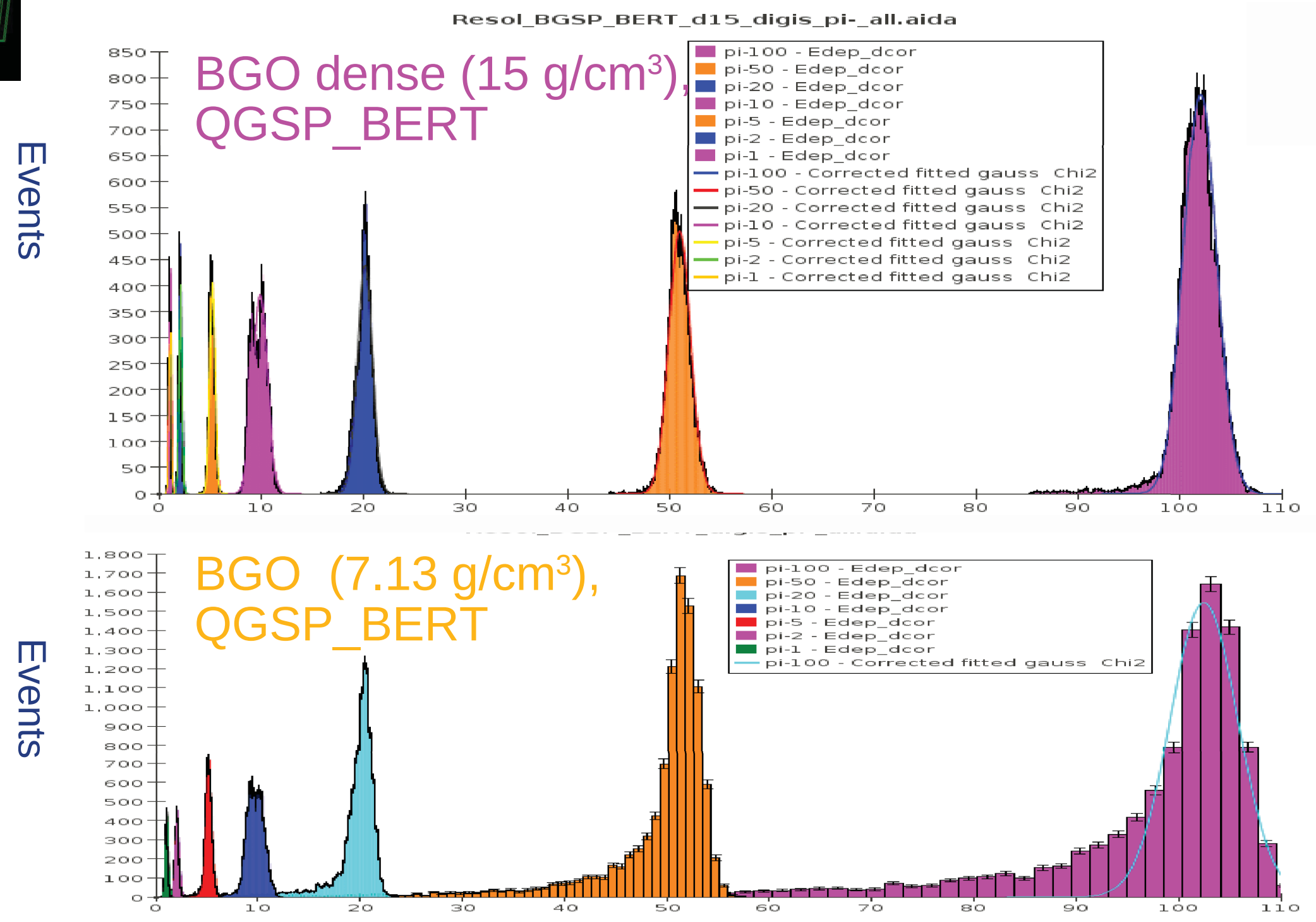

Energy in GeV after dual read out correction 

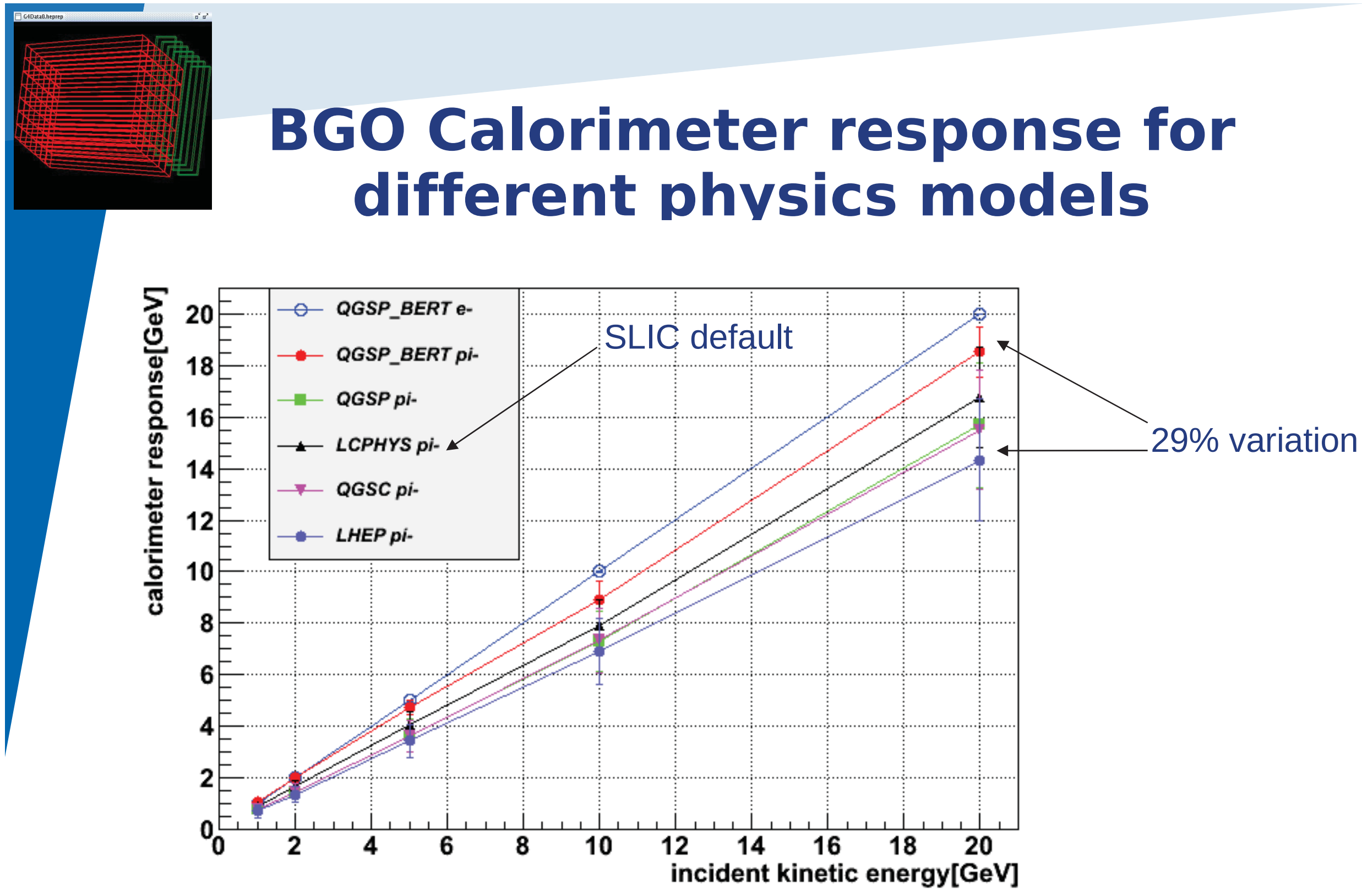

Particles produced within the calorimeter!

No threshold! $\rightarrow$ all energy deposition are added up 


\section{BGO relative width of energy response to charged pions for different physics lists}

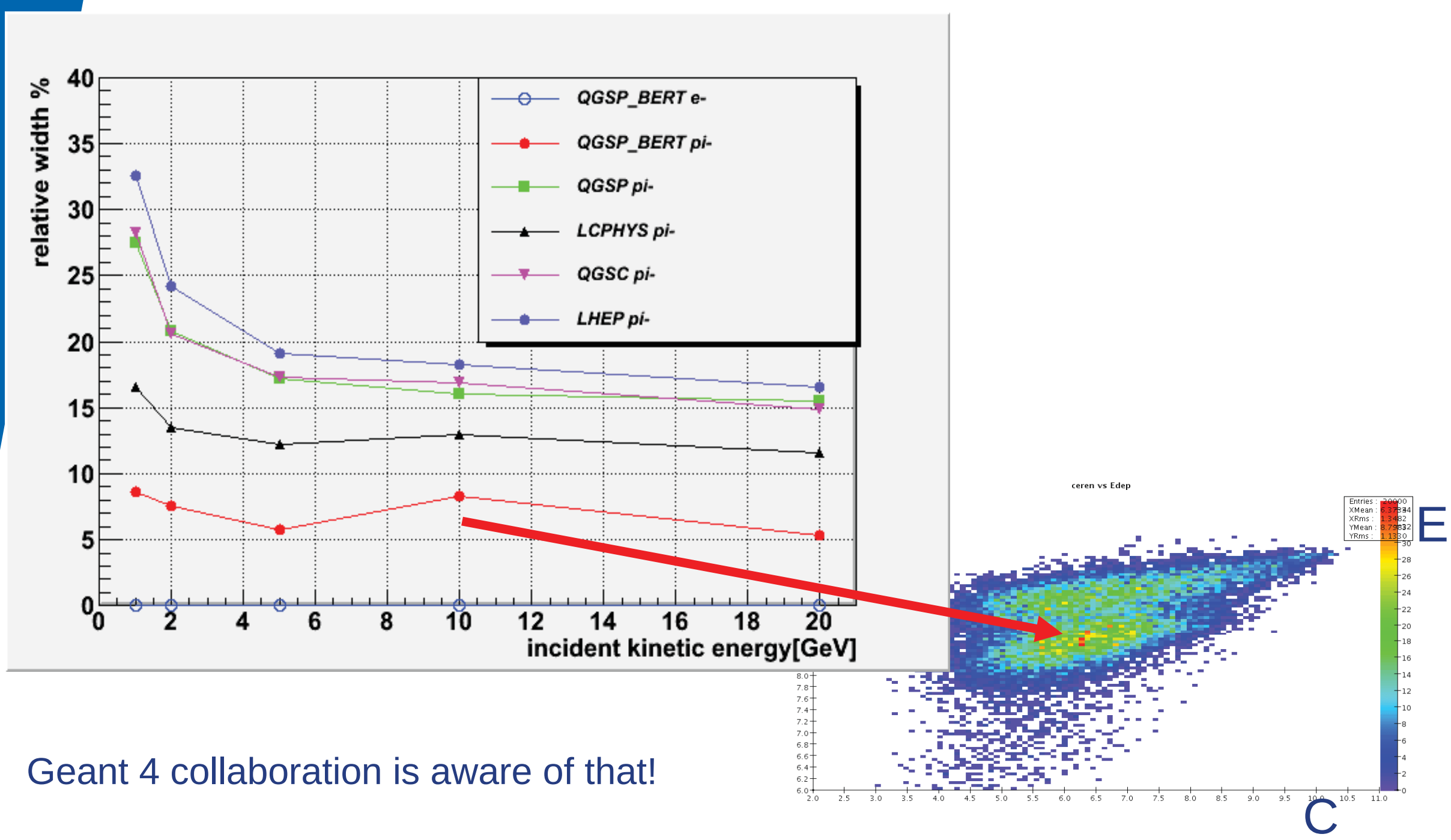




\section{$100 \mathrm{GeV} \pi$ leakage for BGO/PbW04/ BGO dense}

The leakage energy
fluctuates and the
fractional fluctuation
increases with energy
until it exceeds the
stochastic term
and sets the limit on the
achievable energy
resolution.
Leakage fluctuations
depend on:
-the starting point of the
hadron shower
(Interaction Depth or ID)
-the extension of the
shower

pi-100 - Edep

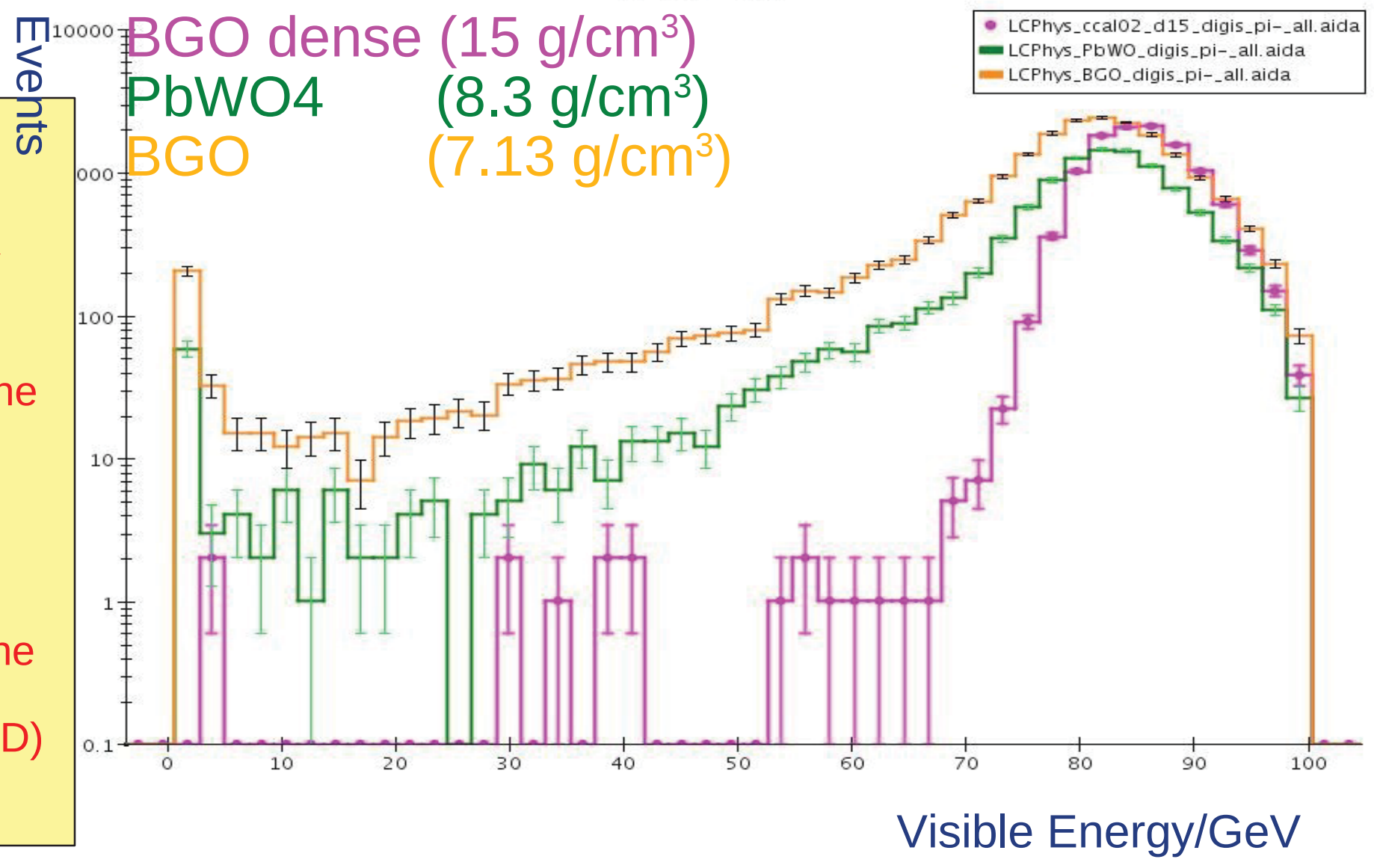

Leakage is of particular concern for compact detectors such as SID! 


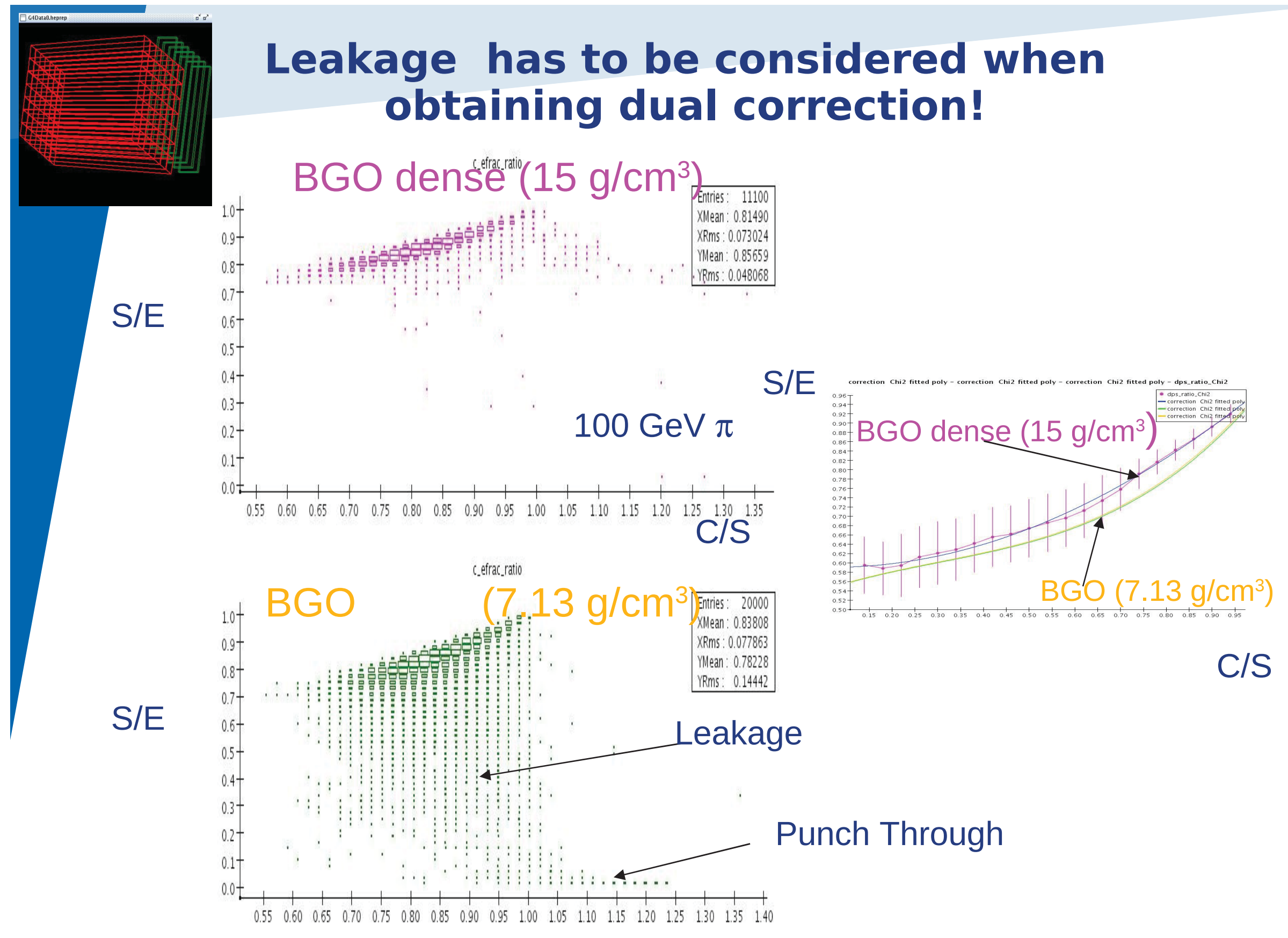

C/S 


\section{Birks attenuation}

Implemented in SLIC,

Available in Geant 4 via Szintillation process

$\frac{d L}{d x}=\frac{S \cdot \frac{d E}{d x}}{1+k B \cdot \frac{d E}{d x}}$

Where:

$\mathrm{kB}=$ Birks constant

$S=$ Scintillation Efficiency

$\frac{d L}{d x}=$ Light Output

BGO: $\mathrm{kB}=6.5 \mu \mathrm{m} / \mathrm{MeV}$

(NIM A439 (2000) 158-166)

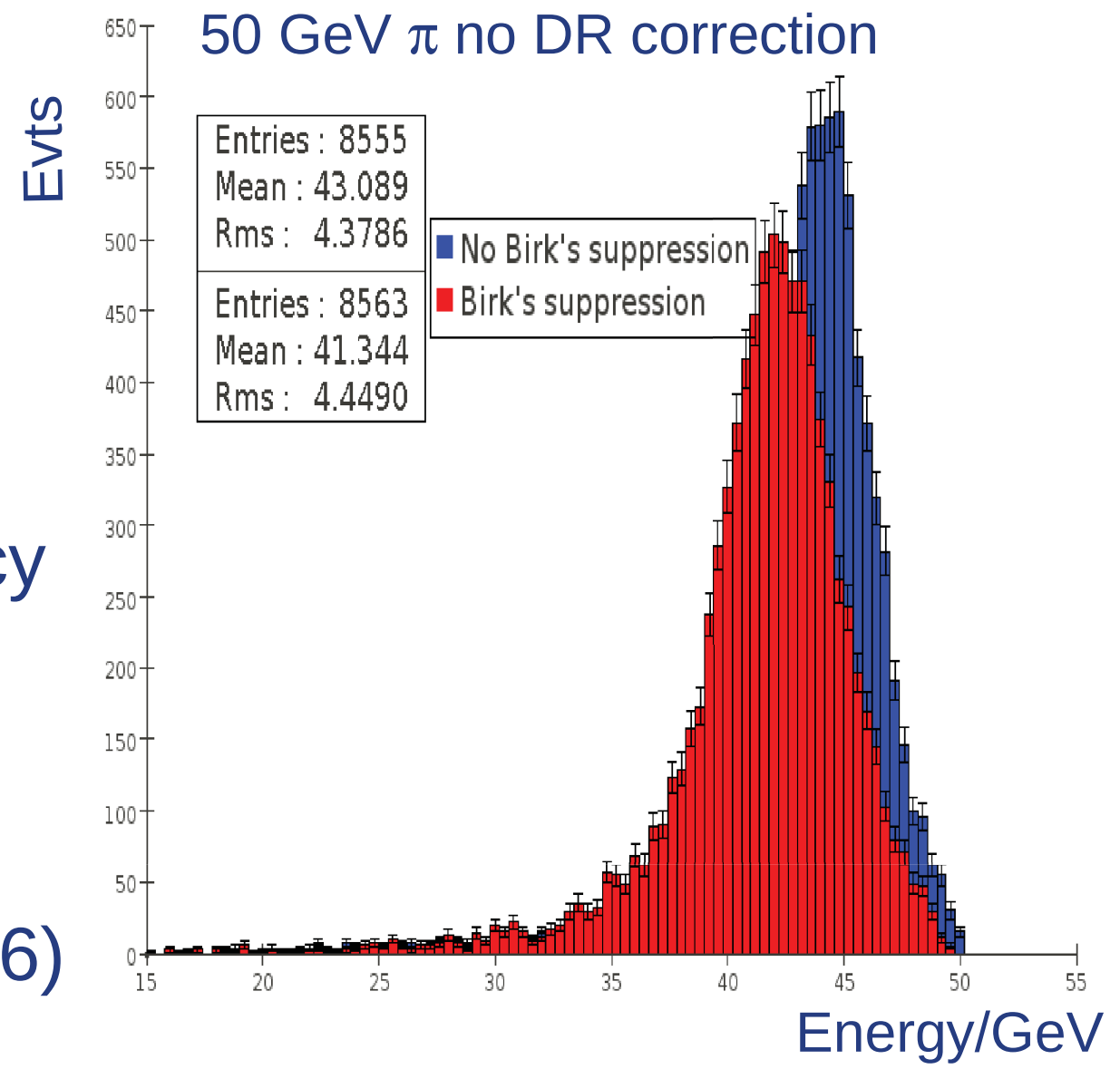




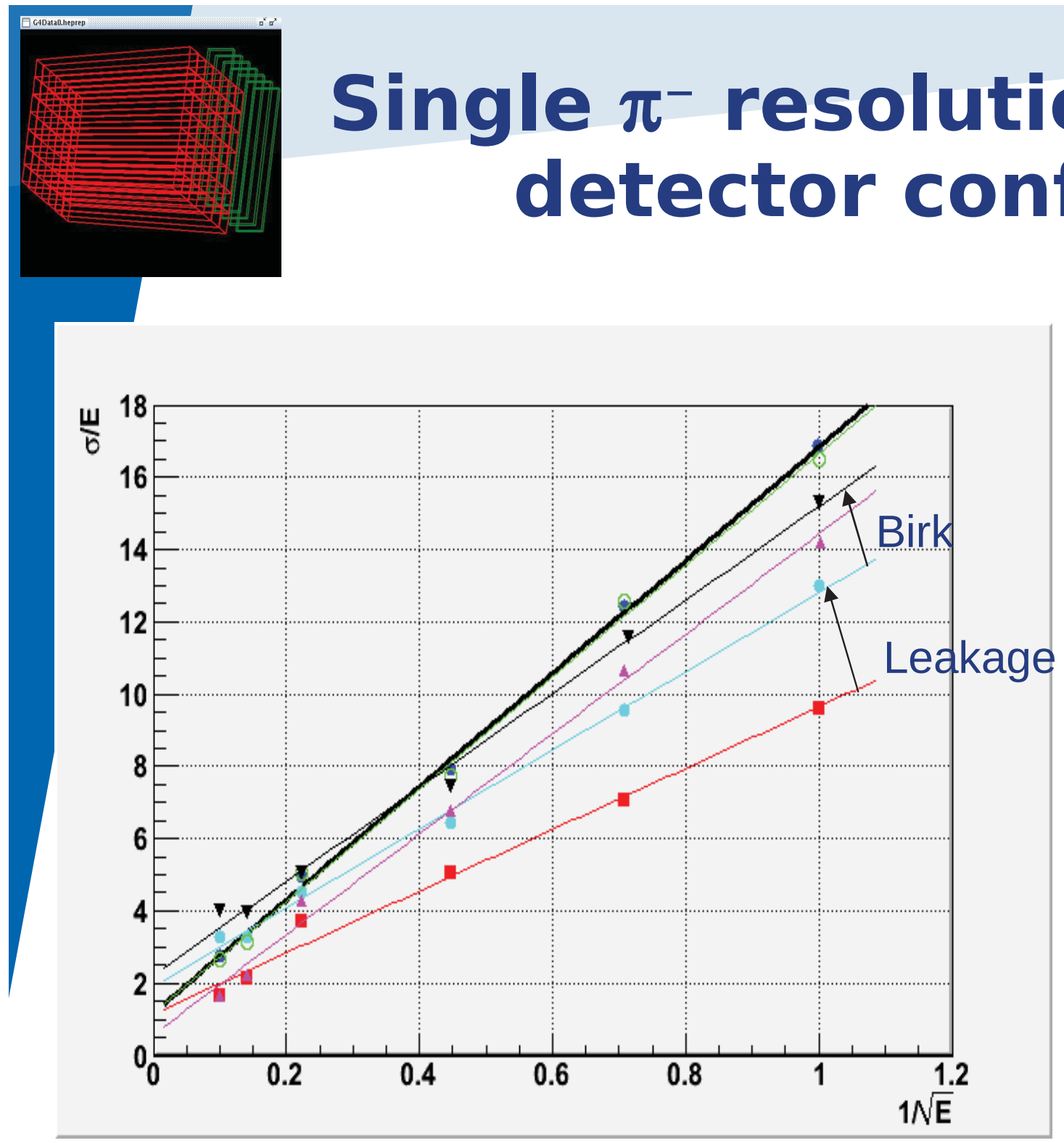

BGO(dense), QGSP BERT: $\sigma(\mathrm{E}) / \mathrm{E}=1.1+8.5 / \mathrm{sqrt}(\mathrm{E}) \%$

BGO, QGSP BERT: $\sigma(E) / E=1.9+10.9 / \operatorname{sqrt}(E) \%$

BGO, QGSP BERT, Birk supr.: $\sigma(\mathrm{E}) / \mathrm{E}=2.23+13.0 / \mathrm{sqrt}(\mathrm{E}) \%$

BGO(dense), LCPhys: $\sigma(E) / E=0.6+13.8 /$ sqrt(E) \%

BGO, LCPhys: (nominal) $\sigma(\mathrm{E}) / \mathrm{E}=1.2+15.6 / \mathrm{sqrt}(\mathrm{E}) \%$

PbWO4, LCPhys: $\sigma(\mathrm{E}) / \mathrm{E}=1.2+15.5 / \mathrm{sqrt}(\mathrm{E}) \%$

Using global dual read out correction $\rightarrow$ can be Improved using energy dependent correction. 


\section{Conclusions}

We have developed a flexible and robust set of tools for simulation of dual readout hadron calorimeters with various geometries, from test-beam to collider detectors.

We have studied in detail the performance of a total absorption dual readout calorimeter, using the Cherenkov-scintillation correlation to correct for the energy depositions undetected or under-detected via scintillation light

We have developed an automatic procedure for derivation of a DR correction from the test beam measurement.

This correction has very slight energy dependence. Even its energy-independent implementation indicate that the energy resolution in the range $10-15 \% / s q r t(E)$ should be achievable.

- The correction determined for single particles works well for collection of particles

- The energy resolution predicted by the full GEANT4 simulation is limited by the modeling imperfections due to transition between the models, and not by the simulated fluctuations of the observed signals in the models themselves 


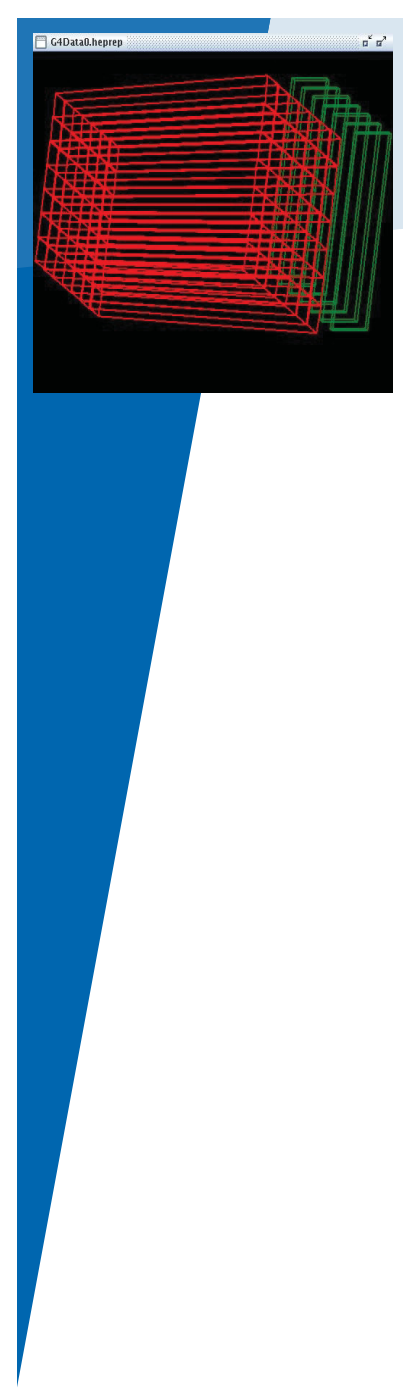

\section{Backup slides}




\section{Motivation}

Lepton Colliders provide a clean environment and aim for high precision measurements complementing discovery machines like the LHC. We don't know what physics scenarios we will finally encounter. We should be ready for all scenarios and aim to build the best possible detector/calorimeter.

Charmonium System Observed Through Inclusive Photons: CB

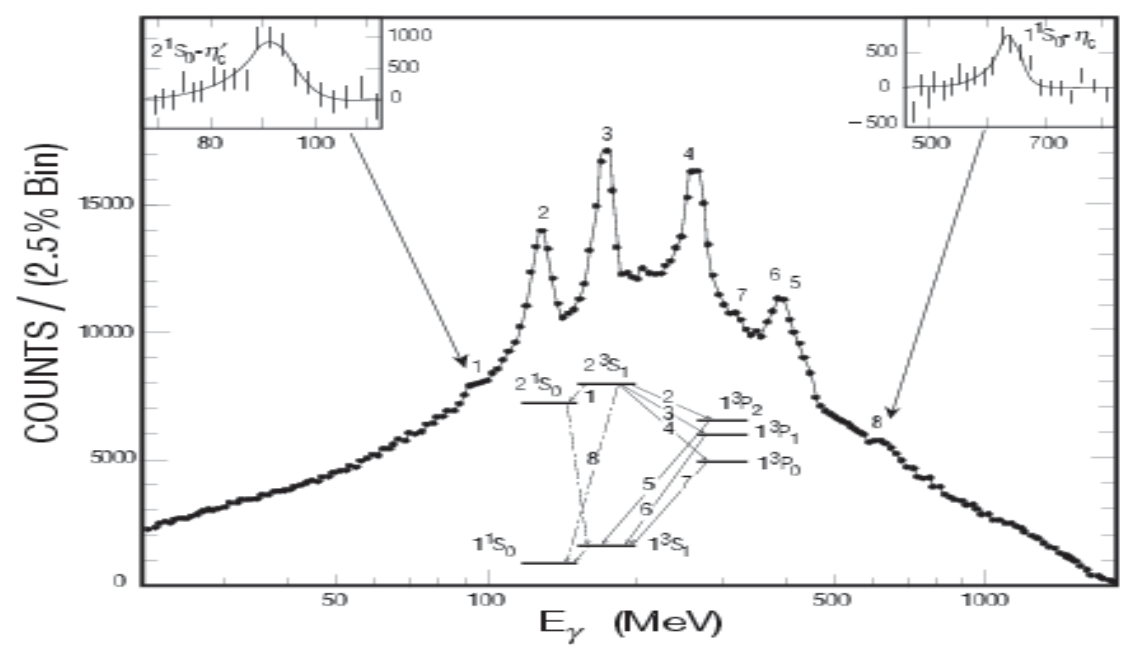

SUSY Breaking with Gravitino

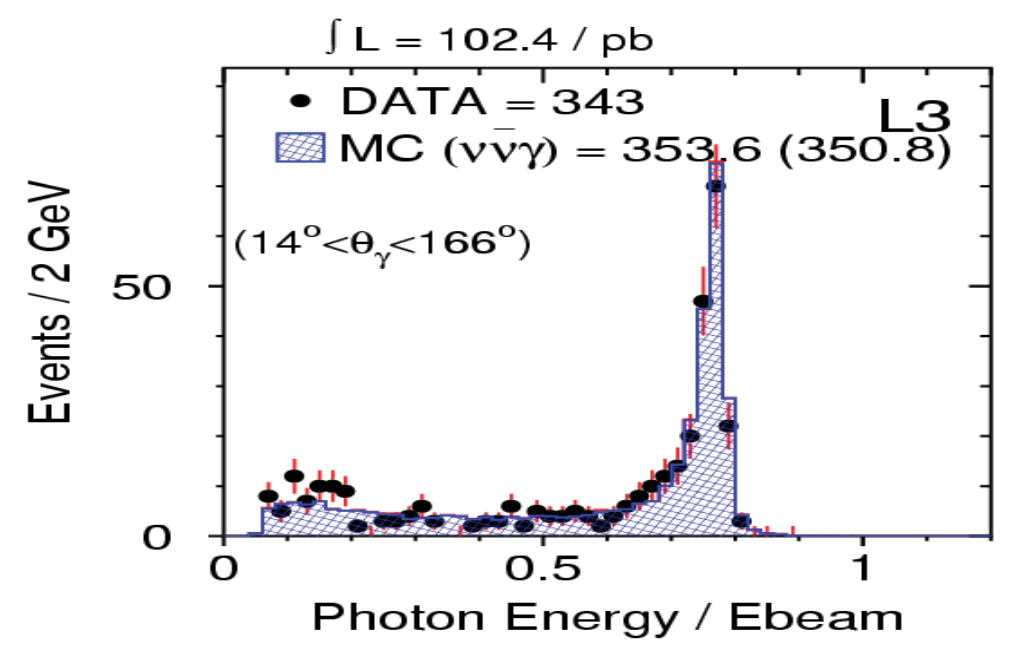




\section{BGO optical properties (II)}
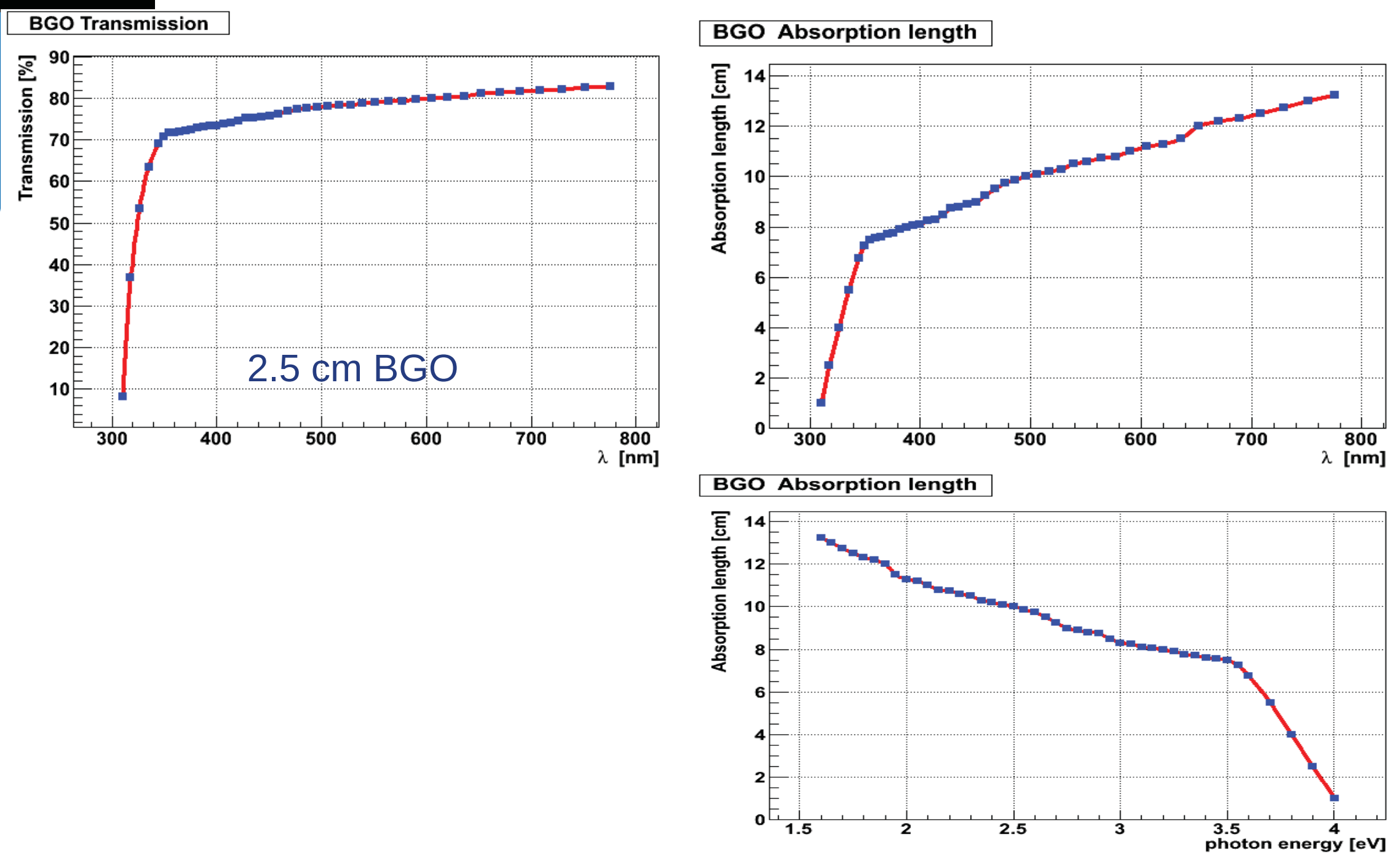


\section{Geant 4: produced Scintillation light}
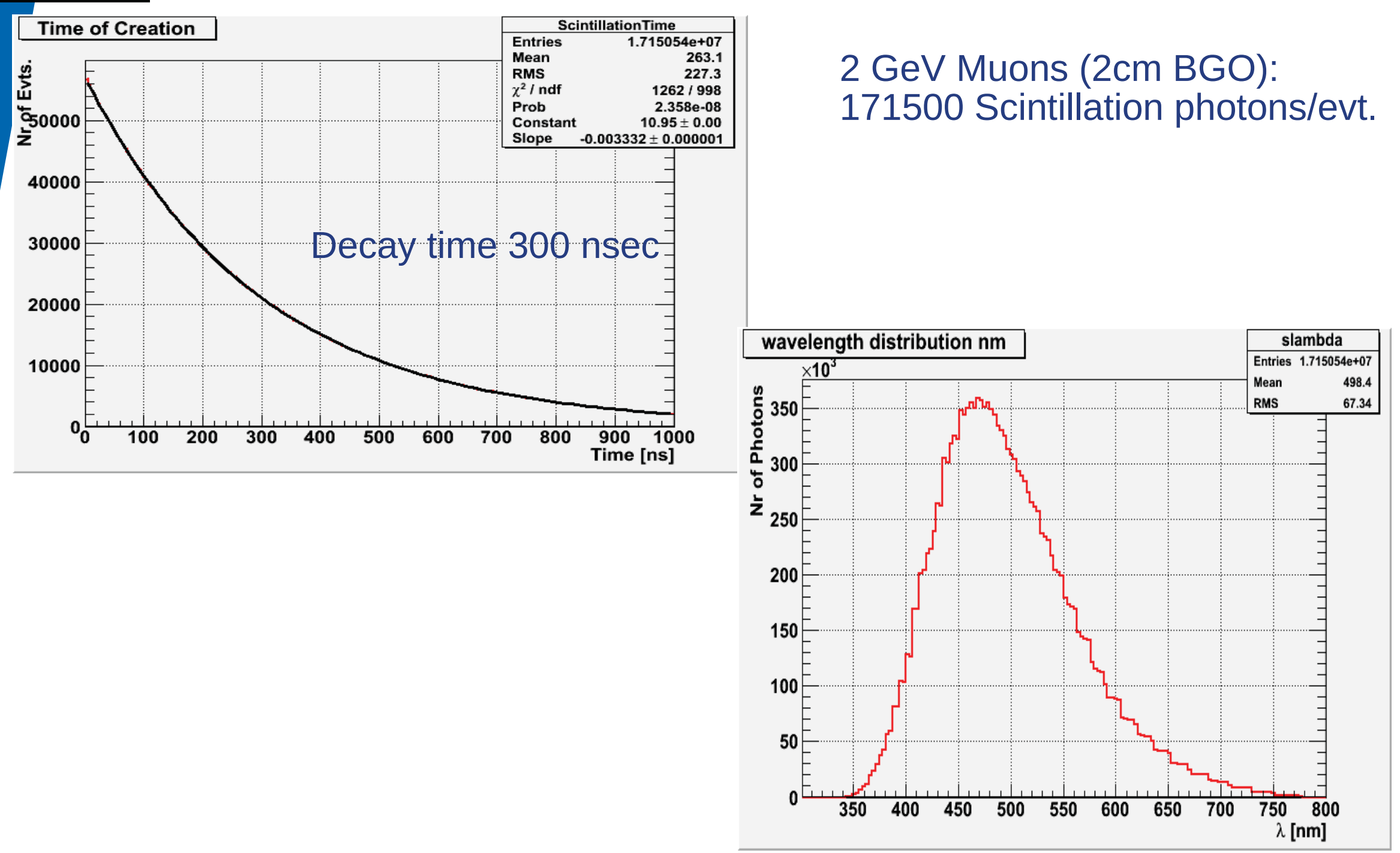

$2 \mathrm{GeV}$ Muons (2cm BGO):

171500 Scintillation photons/evt. 


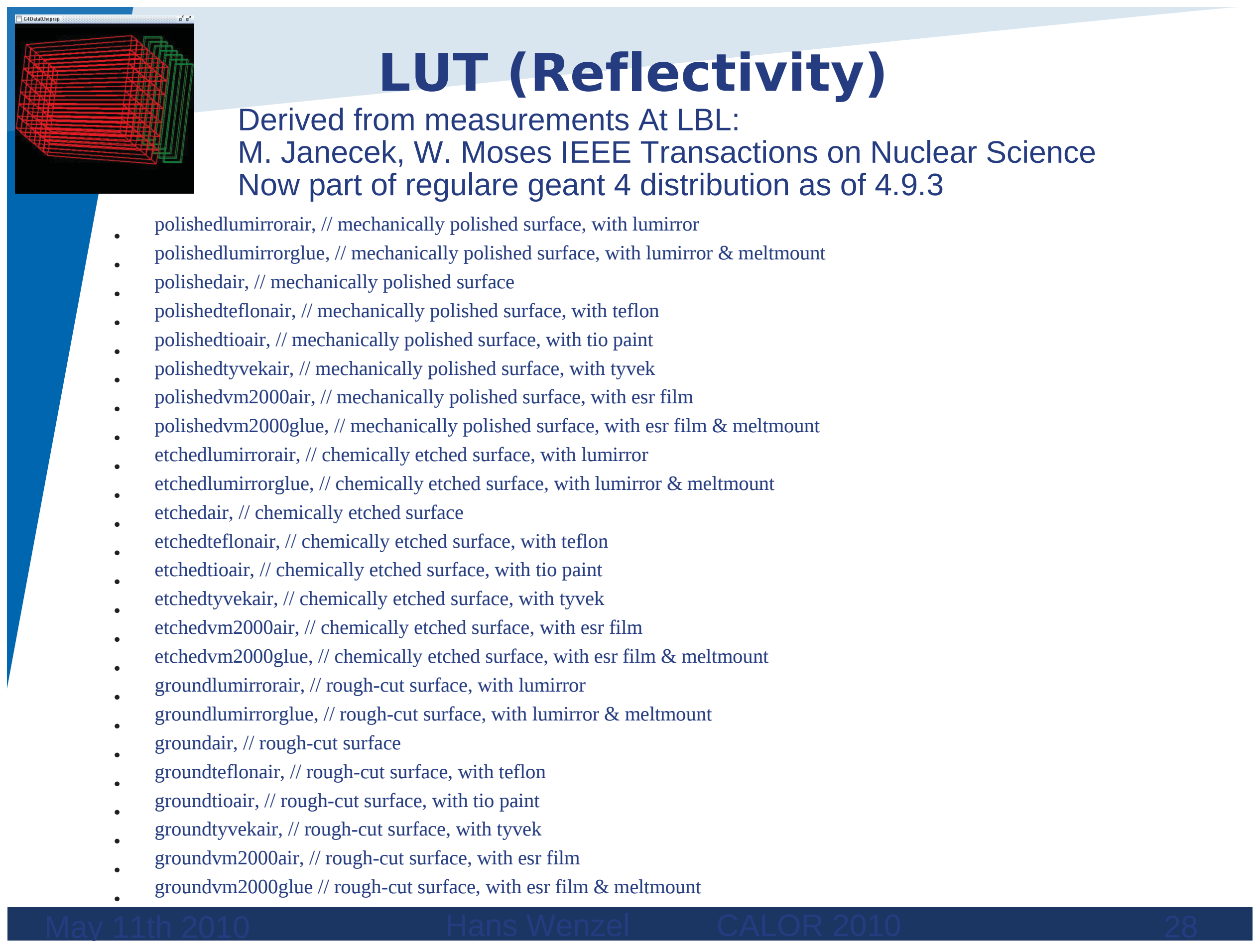




\section{How to use the LUT}

set an environment variable, G4REALSURFACEDATA, to the directory of geant4/data/RealSurface1.0.

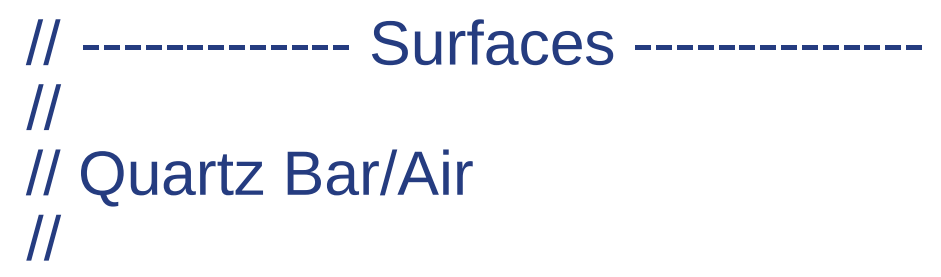

G4LogicalBorderSurface* BGOSurface = new G4LogicalBorderSurface("BGOSurface", BGOBar_phys, expHall_phys, OpBGOSurface); 


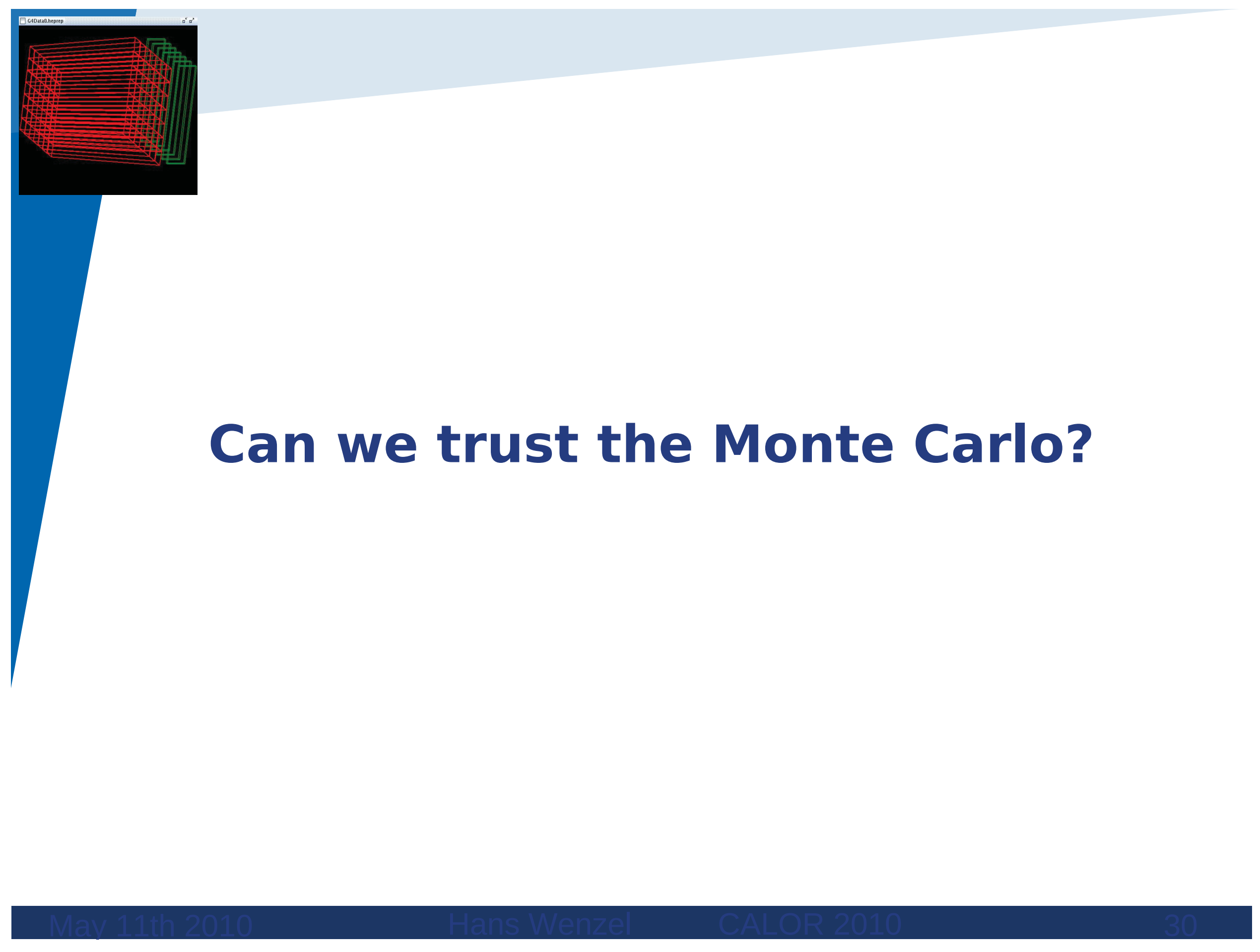




\section{Conclusions}

We have developed a flexible and robust set of tools for simulation of dual readout hadron calorimeters with various geometries, from test-beam to collider detectors. We have studied in details the performance of a total absorption dual readout calorimeter, using the Cherenkov-scintillation correlation to correct for the energy depositions undetected or under-detected via scintillation light

We have developed an automatic procedure for derivation of a DR correction from the test beam measurement.

This correction has very slight energy dependence. Even its energy-independent implementation indicate that the energy resolution in the range $10-15 \% / \mathrm{sqrt}(\mathrm{E})$ should be achievable.

The correction determined for single particles works well for collection of particles Whereas the actual magnitude of the DR correction depends very strongly on the GEANT4 physics model, the predicted performance of the calorimeter is independent of the simulation model

- The energy resolution predicted by the full GEANT4 simulation is limited by the modeling imperfections due to transition between the models, and not by the simulated fluctuations of the observed signals in the models themselves 


\section{Deconvolute Dual read out from leakage}

Leakage must be considered when obtaining the dual read out correction by e.g. requiring the $\pi$ shower to be fully contained. If this is not done correctly leads to overcorrection!

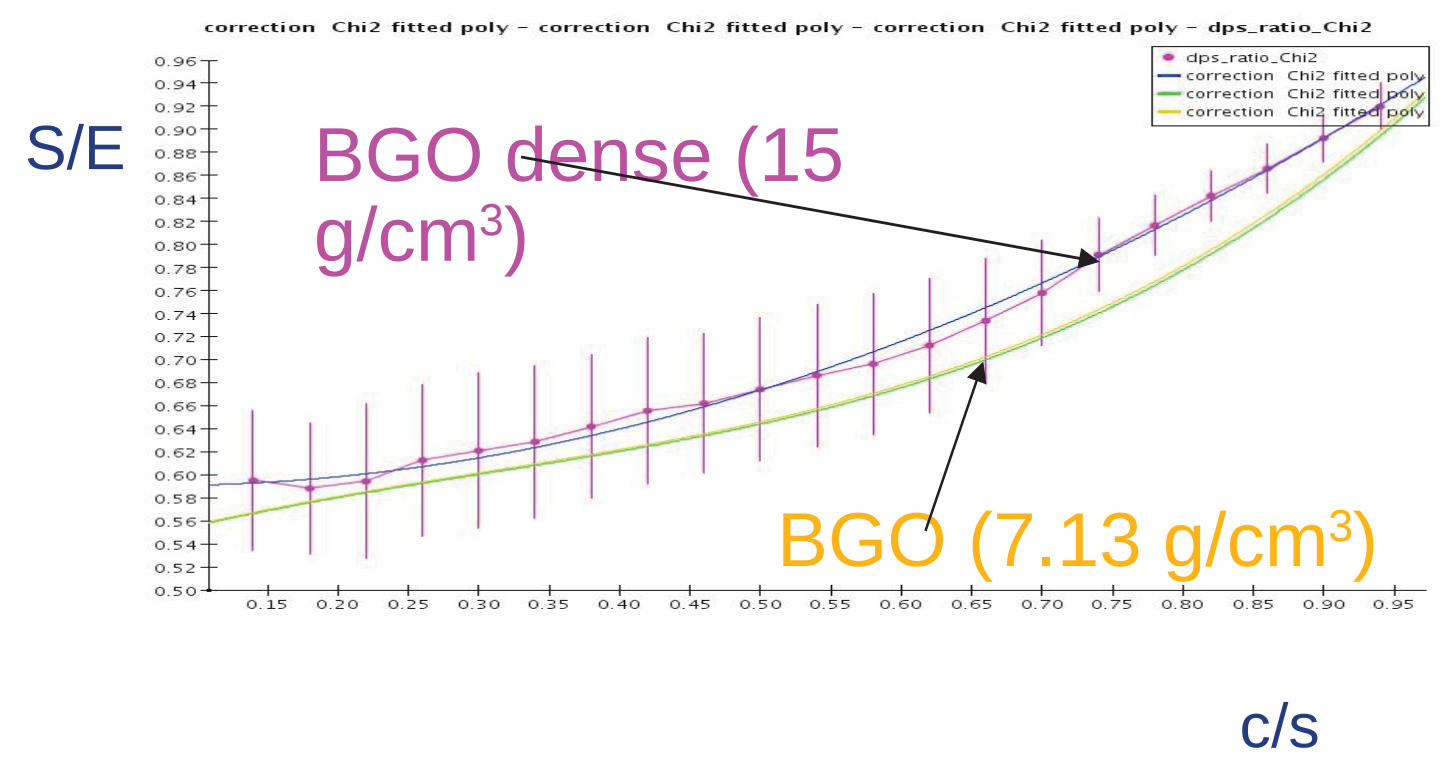

Segmentation can be used to correct for leakage:

Giovanni Pauletta and Anna Driutti have developed an Algorithm to correct for leakage 


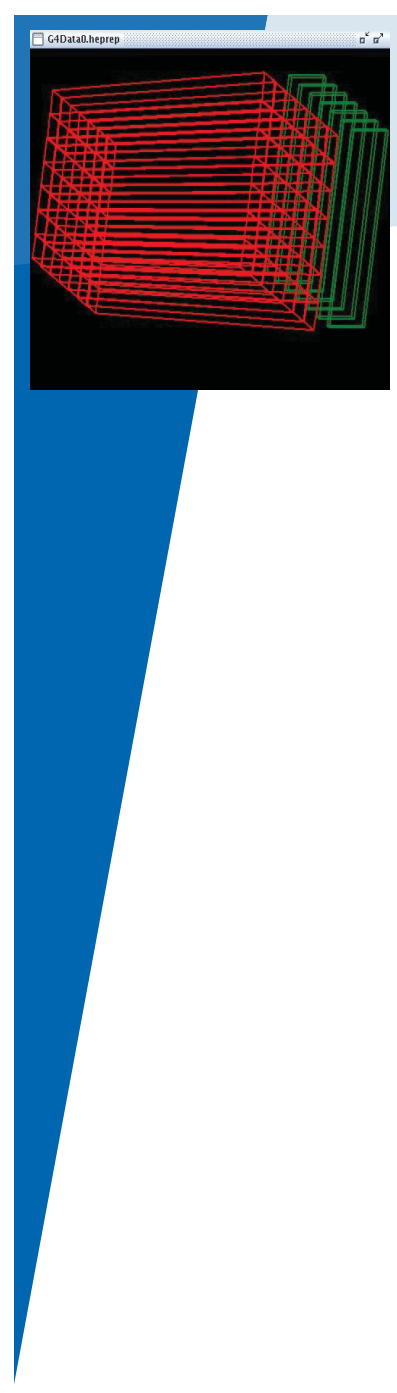

\section{DualCorrection:S/E vs C/S all energies combined}
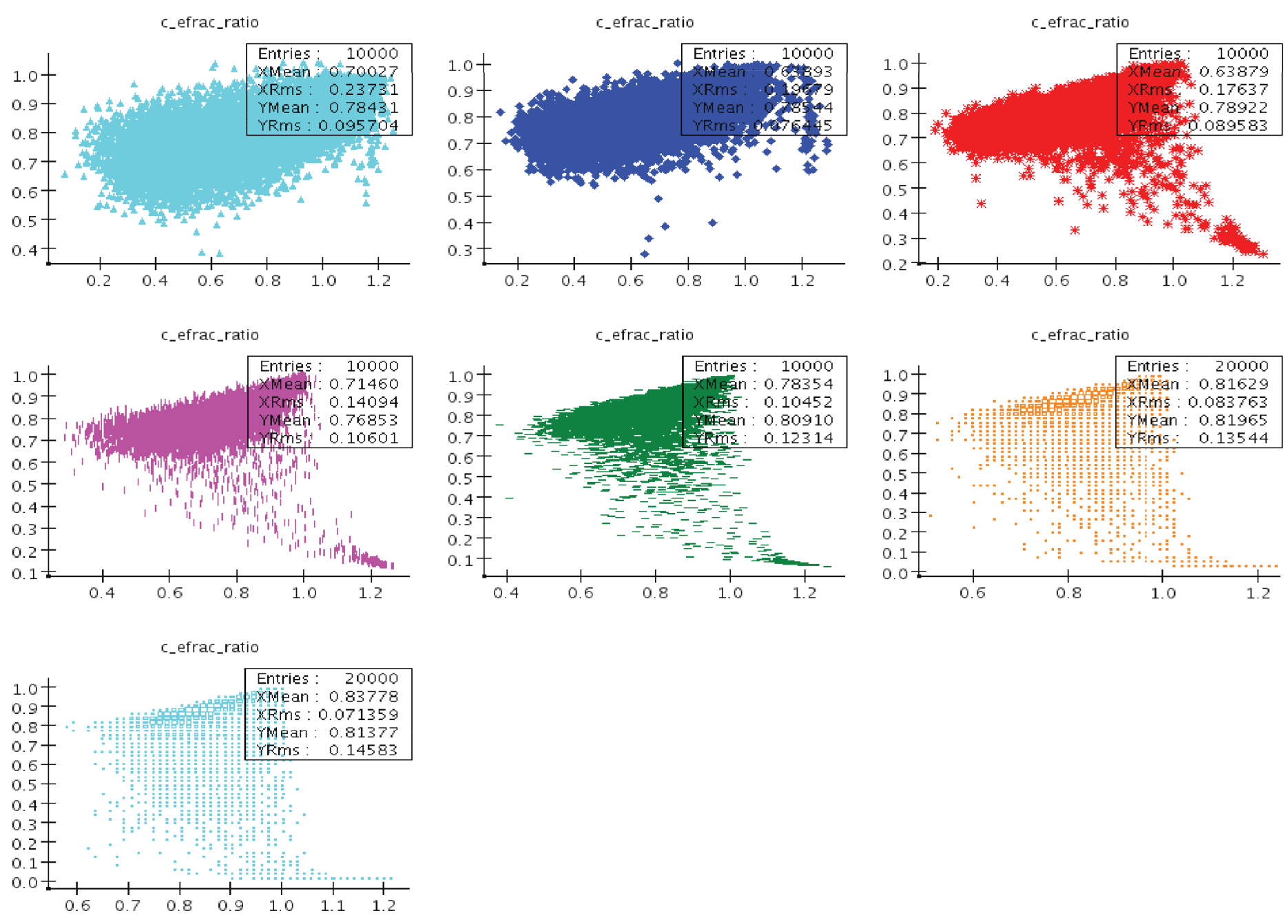


\section{Crystals in a Test Beam}

Single crystal studies:

- Scintillation and Cerenkov light yield

- With different filters

- With different photo detectors

- Position dependence

- Angular dependence

- Angular distribution of Cerenkov light in a shower

- Time profiles

- Compare with detailed Geant 4 simulations.

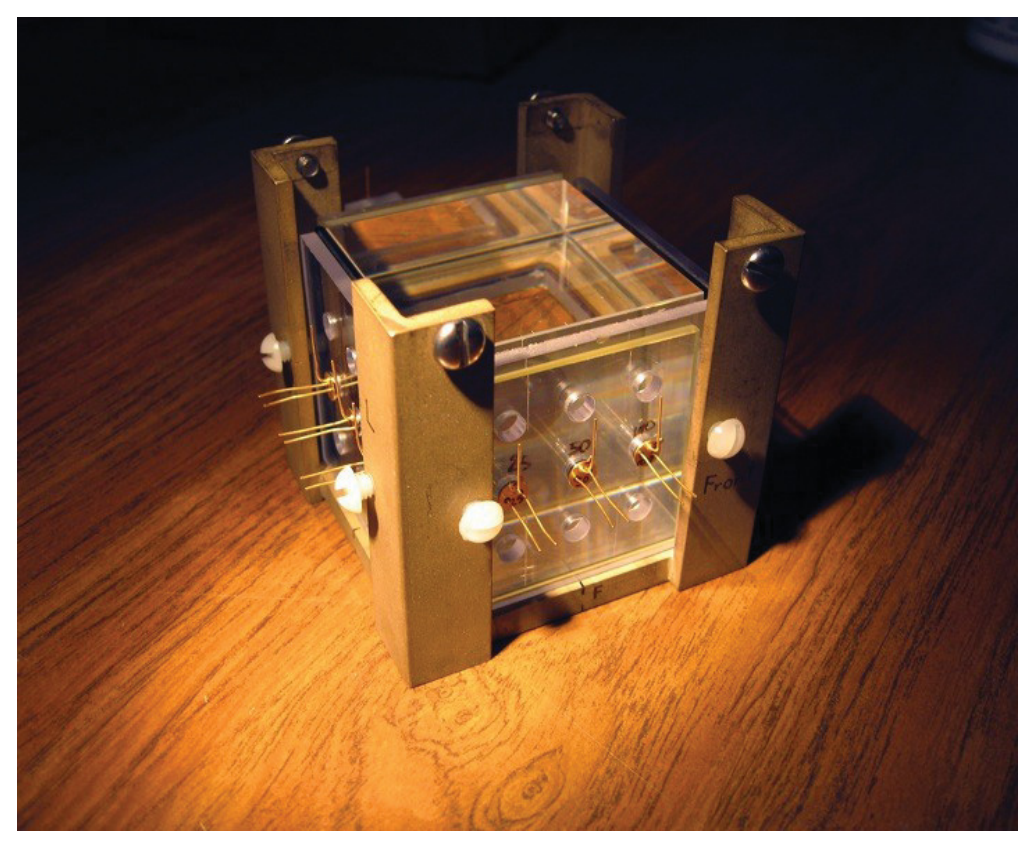




\section{Crystals in a Test Beam}

- $\quad$ CMS EM test module (University of lowa)

- 49 PbWO4 crystals,

photomultipliers, light guides

- $\quad$ Sent over from CERN

- Support structure under construction

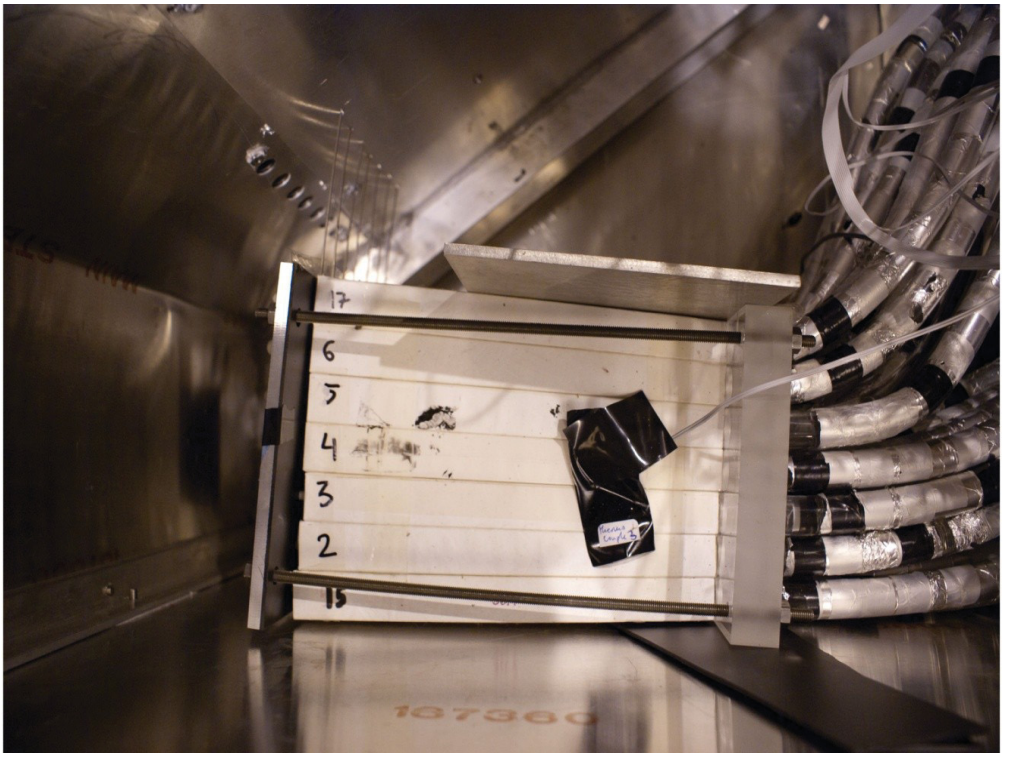

- $\quad$ (Short term) plan:

- Re-assemble the test beam module

- Establish the performance (resolution) for electrons using the oriainal PMT's

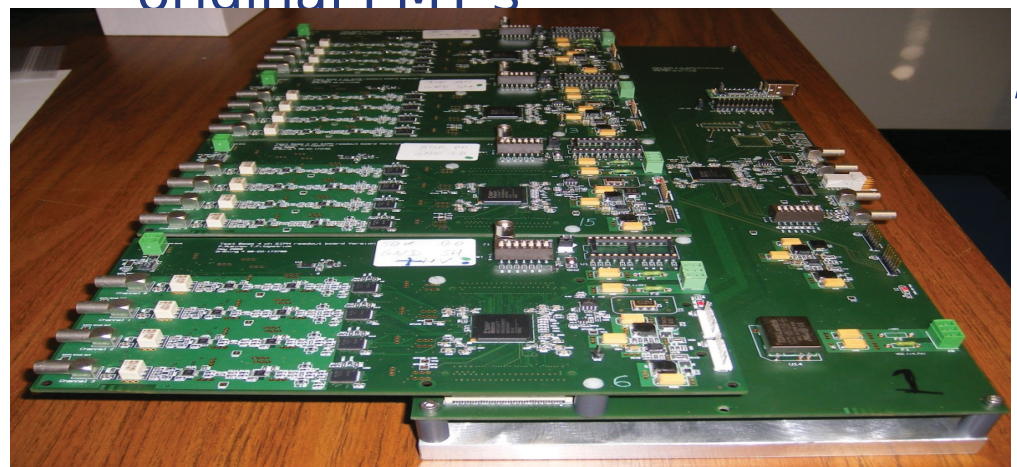

direct

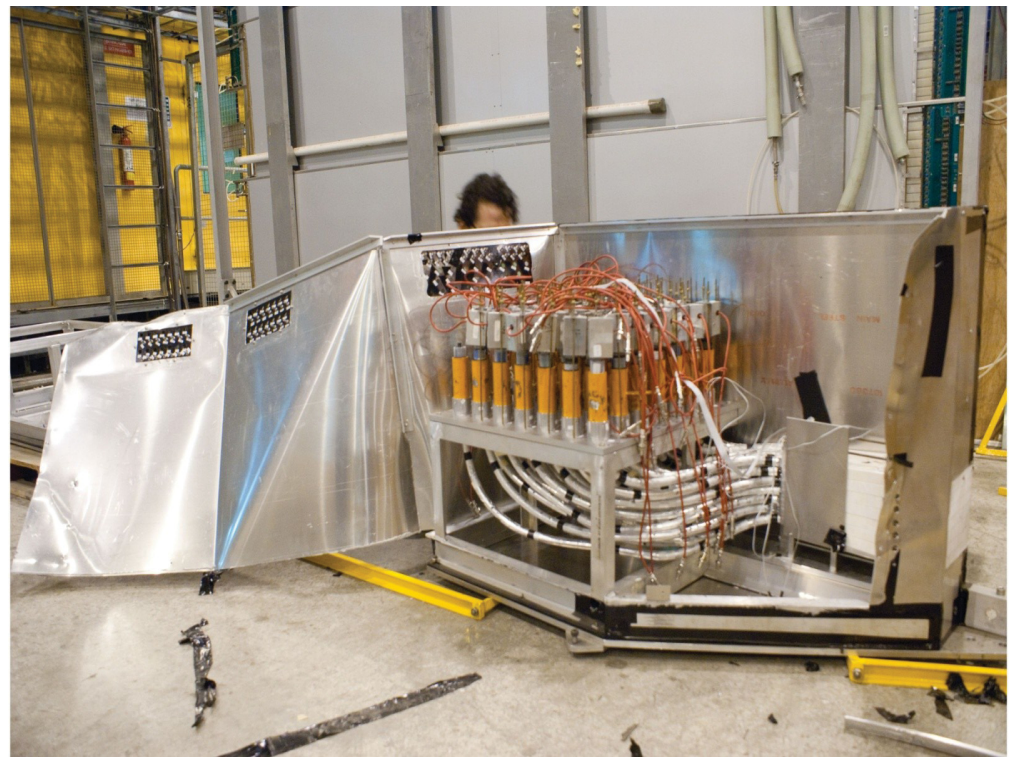




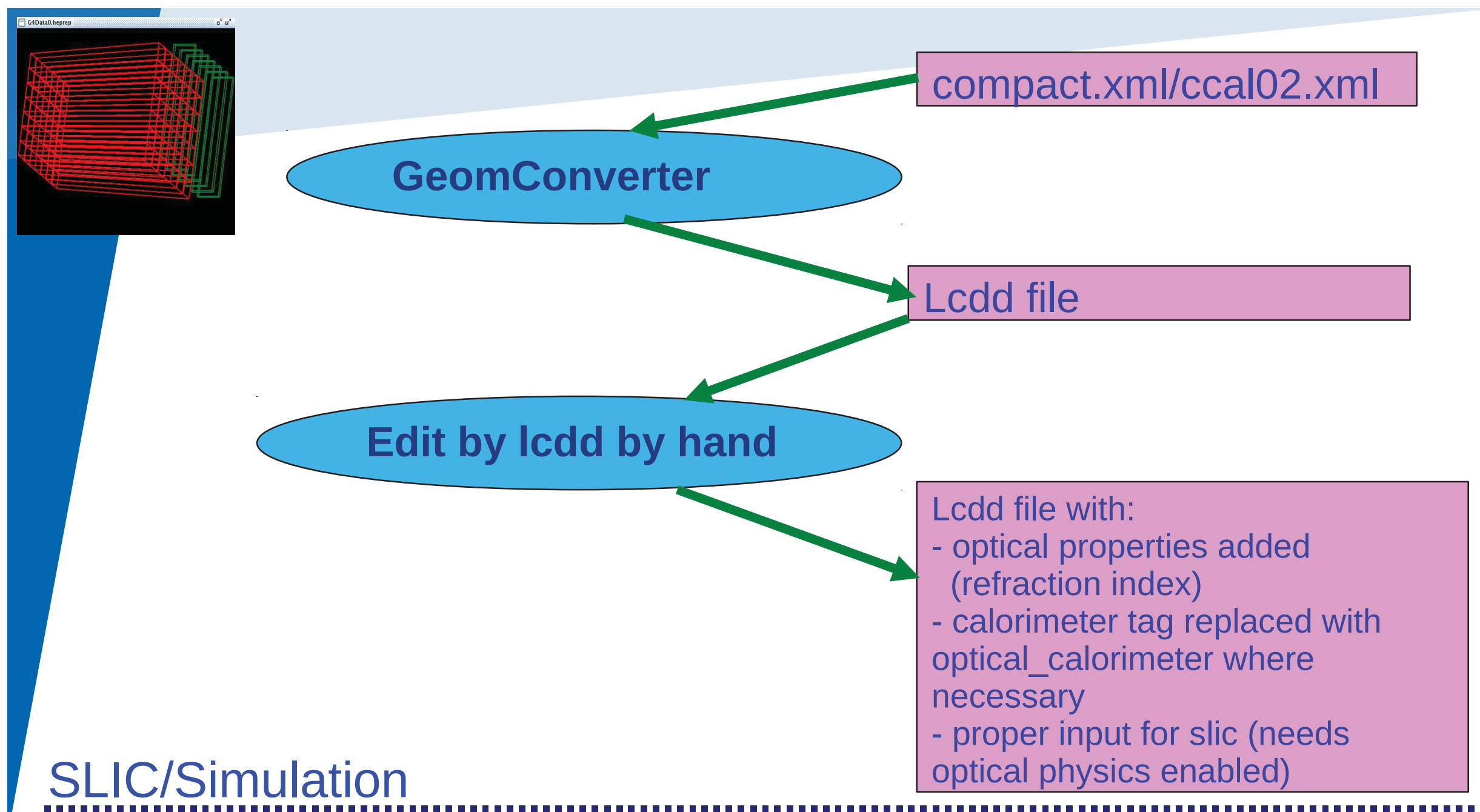

Analysis/Event Display

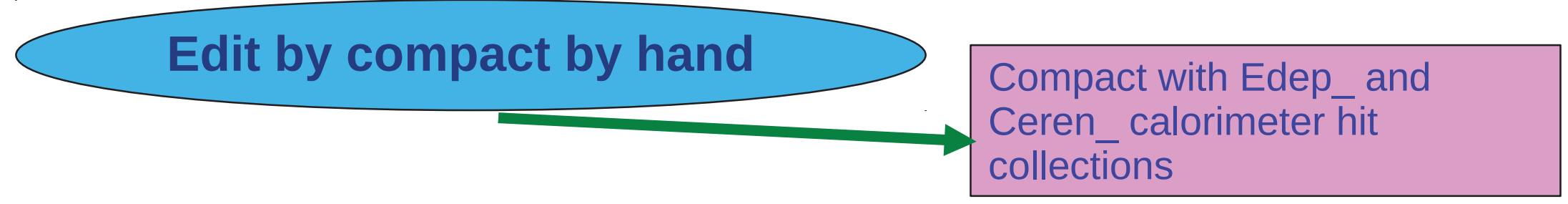

Hans Wenzel 


\section{Width of cerenkov distribution}

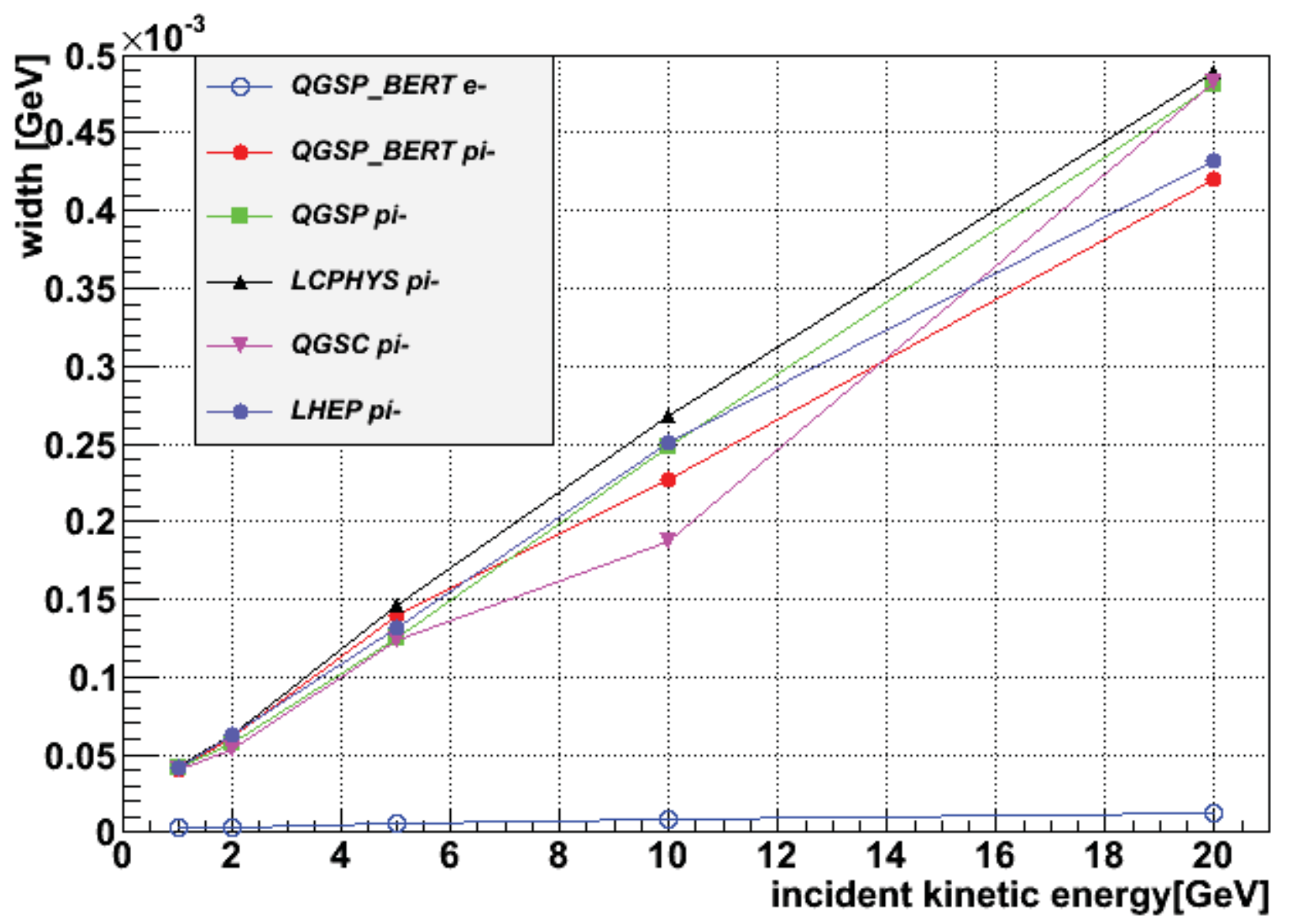




\section{Calorimeter response for different physics models}

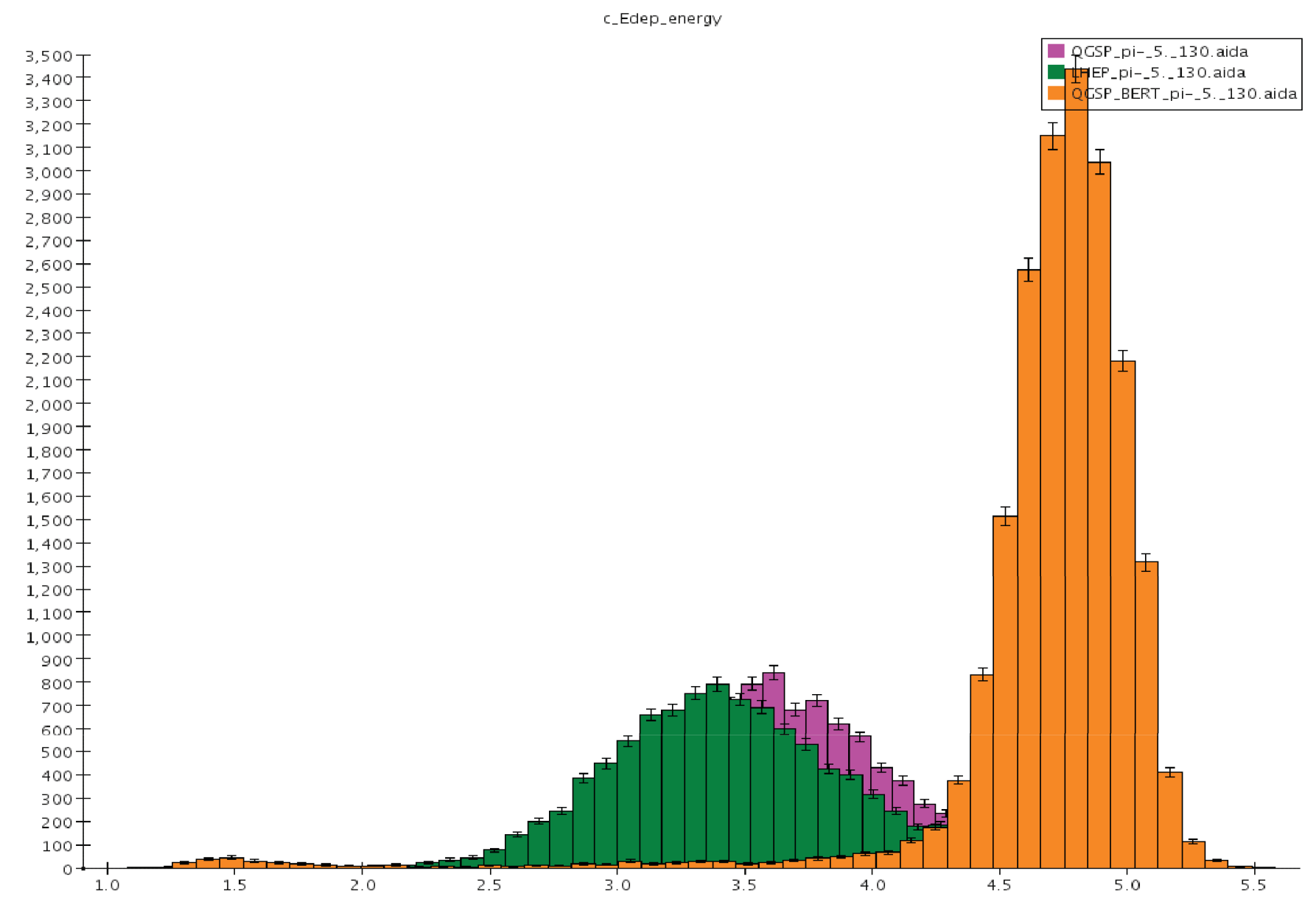




\title{
Spectrum of Cerenkov photons
}

\author{
$n_{\text {photons }}=2 \pi \alpha \sin ^{2} \theta_{c} \cdot \int_{\lambda_{2}}^{\lambda_{1}} \frac{1}{\lambda^{2}} d \lambda$
}

where

$$
\cos \theta_{c}=\frac{1}{\beta n}
$$

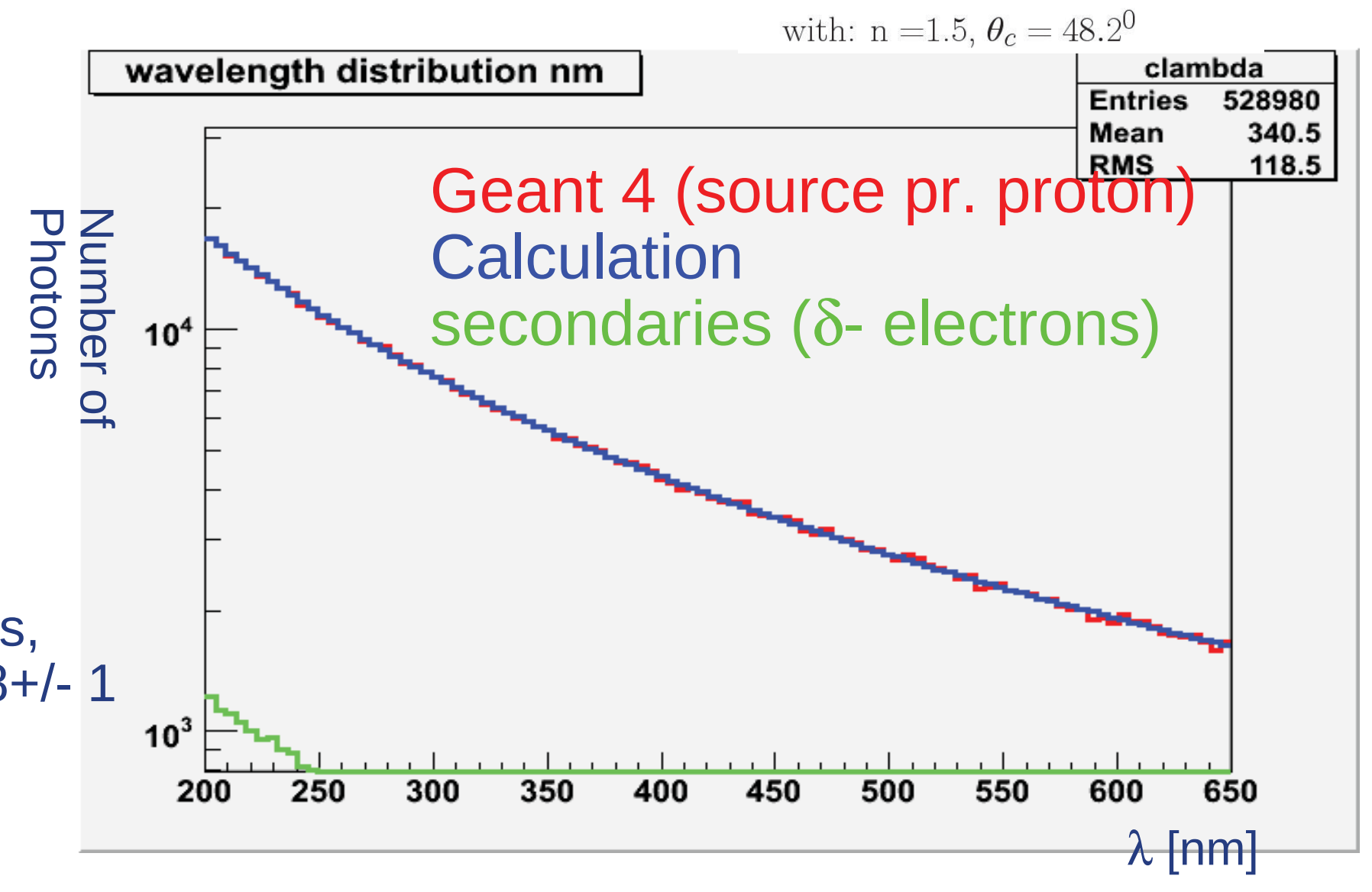

expect $\sim 526$ photons, Geant 4 predicts $528+/-1$

$\lambda[\mathrm{nm}]$ 